\title{
ON THE $x$-COORDINATES OF PELL EQUATIONS WHICH ARE SUMS OF TWO PADOVAN NUMBERS
}

\author{
MAHADI DDAMULIRA
}

\begin{abstract}
Let $\left\{P_{n}\right\}_{n \geq 0}$ be the sequence of Padovan numbers defined by $P_{0}=$ $0, P_{1}=P_{2}=1$, and $P_{n+3}=P_{n+1}+P_{n}$ for all $n \geq 0$. In this paper, we find all positive square-free integers $d$ such that the Pell equations $x^{2}-d y^{2}= \pm 1$, $X^{2}-d Y^{2}= \pm 4$ have at least two positive integer solutions $(x, y)$ and $\left(x^{\prime}, y^{\prime}\right)$, $(X, Y)$ and $\left(X^{\prime}, Y^{\prime}\right)$, respectively, such that each of $x, x^{\prime}, X, X^{\prime}$ is a sum of two Padovan numbers.
\end{abstract}

\section{INTRODUCTION}

Let $\left\{P_{n}\right\}_{n \geq 0}$ be the sequence of Padovan numbers given by

$$
P_{0}=0, P_{1}=1, P_{2}=1 \text {, and } P_{n+3}=P_{n+1}+P_{n} \text { for all } n \geq 0 .
$$

This is sequence $A 000931$ on the Online Encyclopedia of Integer Sequences (OEIS). The first few terms of this sequence are

$$
\left\{P_{n}\right\}_{n \geq 0}=0,1,1,1,2,2,3,4,5,7,9,12,16,21,28,37,49,65,86,114,151, \ldots
$$

Let $d \geq 2$ be a positive integer which is not a square. It is well known that the Pell equations

$$
x^{2}-d y^{2}= \pm 1
$$

and

$$
X^{2}-d Y^{2}= \pm 4
$$

have infinitely many positive integer solutions $(x, y)$ and $(X, Y)$, respectively. By putting $\left(x_{1}, y_{1}\right)$ and $\left(X_{1}, Y_{1}\right)$ for the smallest positive solutions to (1) and (2), respectively, all solutions are of the forms $\left(x_{k}, y_{k}\right)$ and $\left(X_{k}, Y_{k}\right)$ for some positive integer $k$, where

$$
x_{k}+y_{k} \sqrt{d}=\left(x_{1}+y_{1} \sqrt{d}\right)^{k} \quad \text { for all } \quad k \geq 1,
$$

and

$$
\frac{X_{k}+Y_{k} \sqrt{d}}{2}=\left(\frac{X_{1}+Y_{1} \sqrt{d}}{2}\right)^{k} \quad \text { for all } \quad k \geq 1 .
$$

Furthermore, the sequences $\left\{x_{k}\right\}_{k \geq 1}$ and $\left\{X_{k}\right\}_{k \geq 1}$ are binary recurrent. In fact, the following formulae

$$
x_{k}=\frac{\left(x_{1}+y_{1} \sqrt{d}\right)^{k}+\left(x_{1}-y_{1} \sqrt{d}\right)^{k}}{2},
$$

and

$$
X_{k}=\left(\frac{X_{1}+Y_{1} \sqrt{d}}{2}\right)^{k}+\left(\frac{X_{1}-Y_{1} \sqrt{d}}{2}\right)^{k}
$$

2010 Mathematics Subject Classification. 11B39, 11D45, 11D61, 11J86.

Key words and phrases. Padovan numbers; Pell equations; linear forms in logarithms; reduction method. 
hold for all positive integers $k$.

Recently, Bravo et al. [1, 2] studied the Diophantine equation

$$
x_{\ell}=T_{m}+T_{n},
$$

where $x_{\ell}$ are the $x$-coordinates of the solutions of the Pell equation (1) for some positive integer $\ell$ and $\left\{T_{n}\right\}_{n \geq 0}$ is the sequence of Tribonacci numbers given by $T_{0}=0, T_{1}=1=T_{2}$ and $T_{n+3}=T_{n+2}+T_{n+1}+T_{n}$ for all $n \geq 0$. They proved that for each square free integer $d \geq 2$, there is at most one positive integer $\ell$ such that $x_{\ell}$ admits the representation (3) for some nonnegative integers $0 \leq m \leq n$, except for $d \in\{2,3,5,15,26\}$. Furthermore, they explicitly stated all the solutions for these exceptional cases.

In the same spirit, Rihane et al. [16] studied the Diophantine equations

$$
x_{n}=P_{m} \quad \text { and } \quad X_{n}=P_{m},
$$

where $x_{n}$ and $X_{n}$ are the $x$-coordinates of the solutions of the Pell equations (1) and (2), respectively, for some positive integers $n$ and $\left\{P_{m}\right\}_{m \geq 0}$ is the sequence of Padovan numbers. They proved that for each square free integer $d \geq 2$, there is at most one positive integer $x$ participating in the Pell equation (1) and one positive integer $X$ participating in the Pell equation (2) that is a Padovan number with a few exceptions of $d$ that they effectively computed. Furthermore, the exceptional cases were $d \in\{2,3,5,6\}$ and $d \in\{5\}$ for the the first and second equations in (4), respectively. Several other related problems have been studied where $x_{l}$ belongs to some interesting positive integer sequences. For example, see [5, 6, 8, 10, 11, 12, 13, 14].

\section{Main Results}

In this paper, we study a problem related to that of Bravo et al.[1, 2] but with the Padovan sequence instead of the Tribonacci sequence. We also extend the results from the Pell equation (1) to the Pell equation (2). In both cases we find that there are only finitely many solutions that we effectively compute.

Since $P_{1}=P_{2}=P_{3}=1$, we discard the situations when $n=1$ and $n=2$ and just count the solutions for $n=3$. Similarly, $P_{4}=P_{5}=2$, we discard the situation when $n=4$ and just count the solutions for $n=5$. The main aim of this paper is to prove the following results.

Theorem 1. For each integer $d \geq 2$ which is not a square, there is at most one positive integer $k$ such that $x_{k}$ admits a representation as

$$
x_{k}=P_{n}+P_{m}
$$

for some nonnegative integers $0 \leq m \leq n$, except when $d \in\{2,3,6,15,110,483\}$ in the +1 case and $d \in\{2,5,10,17\}$ in the -1 case.

Theorem 2. For each integer $d \geq 2$ which is not a square, there is at most one positive integer $k$ such that $X_{k}$ admits a representation as

$$
X_{k}=P_{n}+P_{m}
$$

for some nonnegative integers $0 \leq m \leq n$, except when $d \in\{3,5,21\}$ in the +4 case and $d \in\{2,5\}$ in the -4 case.

For the exceptional values of $d$ listed in Theorem 1 and Theorem 2 , all solutions $(k, n, m)$ are listed at the end of the proof of each result. The main tools used in this paper are the lower bounds for linear forms in logarithms of algebraic numbers and the Baker-Davenport reduction procedure, as well as the elementary properties of Padovan numbers and solutions to Pell equations. 


\section{Preliminary Results}

3.1. The Padovan sequence. Here, we recall some important properties of the Padovan sequence $\left\{P_{n}\right\}_{n \geq 0}$. The characteristic equation

$$
x^{3}-x-1=0
$$

has roots $\alpha, \beta, \gamma=\bar{\beta}$, where

$$
\alpha=\frac{r_{1}+r_{2}}{6}, \quad \beta=\frac{-\left(r_{1}+r_{2}\right)+\sqrt{-3}\left(r_{1}-r_{2}\right)}{12}
$$

and

$$
r_{1}=\sqrt[3]{108+12 \sqrt{69}} \text { and } r_{2}=\sqrt[3]{108-12 \sqrt{69}}
$$

Furthermore, the Binet formula is given by

$$
P_{n}=a \alpha^{n}+b \beta^{n}+c \gamma^{n} \quad \text { for all } \quad n \geq 0,
$$

where

$$
a=\frac{\alpha+1}{(\alpha-\beta)(\alpha-\gamma)}, \quad b=\frac{\beta+1}{(\beta-\alpha)(\beta-\gamma)}, \quad c=\frac{\gamma+1}{(\gamma-\alpha)(\gamma-\beta)}=\bar{b} .
$$

Numerically, the following estimates hold:

$$
\begin{gathered}
1.32<\alpha<1.33 \\
0.86<|\beta|=|\gamma|=\alpha^{-\frac{1}{2}}<0.87 \\
0.72<a<0.73 \\
0.24<|b|=|c|<0.25
\end{gathered}
$$

From (7), (8) and (11), it is easy to see that the contribution the complex conjugate roots $\beta$ and $\gamma$, to the right-hand side of $(9)$, is very small. In particular, setting

$$
e(n):=P_{n}-a \alpha^{n}=b \beta^{n}+c \gamma^{n} \quad \text { then } \quad|e(n)|<\frac{1}{\alpha^{n / 2}}
$$

holds for all $n \geq 1$. Furthermore, by induction, we can prove that

$$
\alpha^{n-2} \leq P_{n} \leq \alpha^{n-1} \quad \text { holds for all } \quad n \geq 4 .
$$

3.2. Linear forms in logarithms. Let $\eta$ be an algebraic number of degree $d$ with minimal primitive polynomial over the integers

$$
a_{0} x^{d}+a_{1} x^{d-1}+\cdots+a_{d}=a_{0} \prod_{i=1}^{d}\left(x-\eta^{(i)}\right),
$$

where the leading coefficient $a_{0}$ is positive and the $\eta^{(i)}$ 's are the conjugates of $\eta$. Then the logarithmic height of $\eta$ is given by

$$
h(\eta):=\frac{1}{d}\left(\log a_{0}+\sum_{i=1}^{d} \log \left(\max \left\{\left|\eta^{(i)}\right|, 1\right\}\right)\right) .
$$

In particular, if $\eta=p / q$ is a rational number with $\operatorname{gcd}(p, q)=1$ and $q>0$, then $h(\eta)=\log \max \{|p|, q\}$. The following are some of the properties of the logarithmic height function $h(\cdot)$, which will be used in the next sections of this paper without reference:

$$
\begin{aligned}
h\left(\eta_{1} \pm \eta_{2}\right) & \leq h\left(\eta_{1}\right)+h\left(\eta_{2}\right)+\log 2, \\
h\left(\eta_{1} \eta_{2}^{ \pm 1}\right) & \leq h\left(\eta_{1}\right)+h\left(\eta_{2}\right), \\
h\left(\eta^{s}\right) & =|s| h(\eta) \quad(s \in \mathbb{Z}) .
\end{aligned}
$$


We start by recalling the result of Bugeaud, Mignotte, and Siksek ([3, Theorem 9.4, pp. 989), which is a modified version of the result of Matveev [15], which is one of our main tools in this paper.

Theorem 3. Let $\eta_{1}, \ldots, \eta_{t}$ be positive real algebraic numbers in a real algebraic number field $\mathbb{K} \subset \mathbb{R}$ of degree $D_{\mathbb{K}}, b_{1}, \ldots, b_{t}$ be nonzero integers, and assume that

$$
\Lambda:=\eta_{1}^{b_{1}} \cdots \eta_{t}^{b_{t}}-1
$$

is nonzero. Then

$$
\log |\Lambda|>-1.4 \times 30^{t+3} \times t^{4.5} \times D_{\mathbb{K}}^{2}\left(1+\log D_{\mathbb{K}}\right)(1+\log B) A_{1} \cdots A_{t},
$$

where

$$
B \geq \max \left\{\left|b_{1}\right|, \ldots,\left|b_{t}\right|\right\}
$$

and

$$
A_{i} \geq \max \left\{D_{\mathbb{K}} h\left(\eta_{i}\right),\left|\log \eta_{i}\right|, 0.16\right\}, \quad \text { for all } \quad i=1, \ldots, t .
$$

3.3. Reduction procedure. During the calculations, we get upper bounds on our variables which are too large, thus we need to reduce them. To do so, we use some results from the theory of continued fractions.

For the treatment of linear forms homogeneous in two integer variables, we use the well-known classical result in the theory of Diophantine approximation.

Lemma 1. Let $\tau$ be an irrational number, $\frac{p_{0}}{q_{0}}, \frac{p_{1}}{q_{1}}, \frac{p_{2}}{q_{2}}, \ldots$ be all the convergents of the continued fraction of $\tau$ and $M$ be a positive integer. Let $N$ be a nonnegative integer such that $q_{N}>M$. Then putting $a(M):=\max \left\{a_{i}: i=0,1,2, \ldots, N\right\}$, the inequality

$$
\left|\tau-\frac{r}{s}\right|>\frac{1}{(a(M)+2) s^{2}},
$$

holds for all pairs $(r, s)$ of positive integers with $0<s<M$.

For a nonhomogeneous linear form in two integer variables, we use a slight variation of a result due to Dujella and Pethő (see [7], Lemma 5a). For a real number $X$, we write ||$X||:=\min \{|X-n|: n \in \mathbb{Z}\}$ for the distance from $X$ to the nearest integer.

Lemma 2. Let $M$ be a positive integer, $\frac{p}{q}$ be a convergent of the continued fraction of the irrational number $\tau$ such that $q>6 M$, and $A, B, \mu$ be some real numbers with $A>0$ and $B>1$. Let further $\varepsilon:=\|\mu q\|-M\|\tau q\|$. If $\varepsilon>0$, then there is no solution to the inequality

$$
0<|u \tau-v+\mu|<A B^{-w},
$$

in positive integers $u, v$ and $w$ with

$$
u \leq M \quad \text { and } \quad w \geq \frac{\log (A q / \varepsilon)}{\log B} .
$$

At various occasions, we need to find a lower bound for linear forms in logarithms with bounded integer coefficients in three and four variables. In this case we use the LLL- algorithm that we describe below. Let $\tau_{1}, \tau_{2}, \ldots \tau_{t} \in \mathbb{R}$ and the linear form

$$
x_{1} \tau_{1}+x_{2} \tau_{2}+\cdots+x_{t} \tau_{t} \quad \text { with } \quad\left|x_{i}\right| \leq X_{i} .
$$

We put $X:=\max \left\{X_{i}\right\}, C>(t X)^{t}$ and consider the integer lattice $\Omega$ generated by

$$
\mathbf{b}_{j}:=\mathbf{e}_{j}+\left\lfloor C \tau_{j}\right\rceil \quad \text { for } \quad 1 \leq j \leq t-1 \quad \text { and } \quad \mathbf{b}_{t}:=\left\lfloor C \tau_{t}\right\rceil \mathbf{e}_{t},
$$

where $C$ is a sufficiently large positive constant. 
Lemma 3. Let $X_{1}, X_{2}, \ldots, X_{t}$ be positive integers such that $X:=\max \left\{X_{i}\right\}$ and $C>(t X)^{t}$ is a fixed sufficiently large constant. With the above notation on the lattice $\Omega$, we consider a reduced base $\left\{\boldsymbol{b}_{i}\right\}$ to $\Omega$ and its associated Gram-Schmidt orthogonalization base $\left\{\boldsymbol{b}_{i}^{*}\right\}$. We set

$c_{1}:=\max _{1 \leq i \leq t} \frac{\left\|\boldsymbol{b}_{1}\right\|}{\left\|\boldsymbol{b}_{i}^{*}\right\|}, \quad \theta:=\frac{\left\|\boldsymbol{b}_{1}\right\|}{c_{1}}, \quad Q:=\sum_{i=1}^{t-1} X_{i}^{2}, \quad$ and $\quad R:=\frac{1}{2}\left(1+\sum_{i=1}^{t} X_{i}\right)$.

If the integers $x_{i}$ are such that $\left|x_{i}\right| \leq X_{i}$, for $1 \leq i \leq t$ and $\theta^{2} \geq Q+R^{2}$, then we have

$$
\left|\sum_{i=1}^{t} x_{i} \tau_{i}\right| \geq \frac{\sqrt{\theta^{2}-Q}-R}{C} .
$$

For the proof and further details, we refer the reader to the book of Cohen. (Proposition 2.3.20 in ([4], pp. 58-63)).

Finally, the following Lemma is also useful. It is Lemma 7 in [9].

Lemma 4. If $r \geqslant 1, H>\left(4 r^{2}\right)^{r}$, and $H>L /(\log L)^{r}$, then

$$
L<2^{r} H(\log H)^{r} .
$$

\section{Proof of Theorem 1}

Let $\left(x_{1}, y_{1}\right)$ be the smallest positive integer solution to the Pell quation (1). We Put

$$
\delta:=x_{1}+y_{1} \sqrt{d} \text { and } \sigma=x_{1}-y_{1} \sqrt{d} .
$$

From which we get that

$$
\delta \cdot \sigma=x_{1}^{2}-d y_{1}^{2}=: \epsilon, \quad \text { where } \quad \epsilon \in\{ \pm 1\} .
$$

Then

$$
x_{k}=\frac{1}{2}\left(\delta^{k}+\sigma^{k}\right) .
$$

Since $\delta \geq 1+\sqrt{2}$, it follows that the estimate

$$
\frac{\delta^{k}}{\alpha^{4}} \leq x_{k} \leq \delta^{k} \quad \text { holds for all } \quad k \geq 1 .
$$

We assume that $\left(k_{1}, n_{1}, m_{1}\right)$ and $\left(k_{2}, n_{2}, m_{2}\right)$ are triples of integers such that

$$
x_{k_{1}}=P_{n_{1}}+P_{m_{1}} \quad \text { and } \quad x_{k_{2}}=P_{n_{2}}+P_{m_{2}}
$$

We asuume that $1 \leq k_{1}<k_{2}$. We also assume that $3 \leq m_{i}<n_{i}$ for $i=1,2$. We set $(k, n, m):=\left(k_{i}, n_{i}, m_{i}\right)$, for $i=1,2$. Using the inequalities (13) and (20), we get from (21) that

$$
\frac{\delta^{k}}{\alpha^{4}} \leq x_{k}=P_{n}+P_{m} \leq 2 \alpha^{n-1} \quad \text { and } \quad \alpha^{n-2} \leq P_{n}+P_{m}=x_{k} \leq \delta^{k} .
$$

The above inequalities give

$$
(n-2) \log \alpha<k \log \delta<(n+3) \log \alpha+\log 2 .
$$

Dividing through by $\log \alpha$ and setting $c_{2}:=1 / \log \alpha$, we get that

$$
-2<c_{2} k \log \delta-n<3+c_{2} \log 2,
$$

and since $\alpha^{3}>2$, we get

$$
\left|n-c_{2} k \log \delta\right|<6
$$


Furthermore, $k<n$, for if not, we would then get that

$$
\delta^{n} \leq \delta^{k}<2 \alpha^{n+3}, \quad \text { implying } \quad\left(\frac{\delta}{\alpha}\right)^{n}<2 \alpha^{3},
$$

which is false since $\delta \geq 1+\sqrt{2}, 1.32<\alpha<1.33$ (by (11)) and $n \geq 4$.

Besides, given that $k_{1}<k_{2}$, we have by (13) and (21) that

$$
\alpha^{n_{1}-2} \leq P_{n_{1}} \leq P_{n_{1}}+P_{m_{1}}=x_{k_{1}}<x_{k_{2}}=P_{n_{2}}+P_{m_{2}} \leq 2 P_{n_{2}}<2 \alpha^{n_{2}-1} .
$$

Thus, we get that

$$
n_{1}<n_{2}+4 \text {. }
$$

4.1. An inequality for $n$ and $k(\mathbf{I})$. Using the equations $(9)$ and $(19)$ and $(21)$, we get

$$
\frac{1}{2}\left(\delta^{k}+\sigma^{k}\right)=P_{n}+P_{m}=a \alpha^{n}+e(n)+a \alpha^{m}+e(m)
$$

So,

$$
\frac{1}{2} \delta^{k}-a\left(\alpha^{n}+\alpha^{m}\right)=-\frac{1}{2} \sigma^{k}+e(n)+e(m),
$$

and by $(12)$, we have

$$
\begin{aligned}
\left|\delta^{k}(2 a)^{-1} \alpha^{-n}\left(1+\alpha^{m-n}\right)^{-1}-1\right| \leq & \frac{1}{2 \delta^{k} a\left(\alpha^{n}+\alpha^{m}\right)}+\frac{2|b|}{\alpha^{n / 2} a\left(\alpha^{n}+\alpha^{m}\right)} \\
& +\frac{2|b|}{\alpha^{m / 2} a\left(\alpha^{n}+\alpha^{m}\right)} \\
\leq & \frac{1}{a \alpha^{n}}\left(\frac{1}{2 \delta^{k}}+\frac{2|b|}{\alpha^{n / 2}}+\frac{2|b|}{\alpha^{m / 2}}\right)<\frac{1.5}{\alpha^{n}} .
\end{aligned}
$$

Thus, we have

$$
\left|\delta^{k}(2 a)^{-1} \alpha^{-n}\left(1+\alpha^{m-n}\right)^{-1}-1\right|<\frac{1.5}{\alpha^{n}} .
$$

Put

$$
\Lambda_{1}:=\delta^{k}(2 a)^{-1} \alpha^{-n}\left(1+\alpha^{m-n}\right)^{-1}-1
$$

and

$$
\Gamma_{1}:=k \log \delta-\log (2 a)-n \log \alpha-\log \left(1+\alpha^{m-n}\right) .
$$

Since $\left|\Lambda_{1}\right|=\left|e^{\Gamma_{1}}-1\right|<\frac{1}{2}$ for $n \geq 4$ (because $1.5 / \alpha^{4}<1 / 2$ ), since the inequality $|y|<2\left|e^{y}-1\right|$ holds for all $y \in\left(-\frac{1}{2}, \frac{1}{2}\right)$, it follows that $e^{\left|\Gamma_{1}\right|}<2$ and so

$$
\left|\Gamma_{1}\right|<e^{\left|\Gamma_{1}\right|}\left|e^{\Gamma_{1}}-1\right|<\frac{3}{\alpha^{n}} .
$$

Thus, we get that

$$
\left|k \log \delta-\log (2 a)-n \log \alpha-\log \left(1+\alpha^{m-n}\right)\right|<\frac{3}{\alpha^{n}} .
$$

We apply Theorem 3 on the left-hand side of (24) with the data:

$$
\begin{gathered}
t:=4, \quad \eta_{1}:=\delta, \quad \eta_{2}:=2 a, \quad \eta_{3}:=\alpha, \eta_{4}:=1+\alpha^{m-n}, \\
b_{1}:=k, \quad b_{2}:=-1, \quad b_{3}:=-n, \quad b_{4}:=-1 .
\end{gathered}
$$

Furthermore, we take the number field $\mathbb{K}=\mathbb{Q}(\sqrt{d}, \alpha)$ which has degree $D=6$. Since $\max \{1, k, n\} \leq n$, we take $D_{\mathbb{K}}=n$. First we note that the left-hand side of (24) is non-zero, since otherwise,

$$
\delta^{k}=2 a\left(\alpha^{n}+\alpha^{m}\right) .
$$


The left-hand side belongs to the quadratic field $\mathbb{Q}(\sqrt{d})$ while the right-hand side belongs to the cubic field $\mathbb{Q}(\alpha)$. These fields only intersect when both sides are rational numbers. Since $\delta^{k}$ is a positive algebraic integer and a unit, we get that to $\delta^{k}=1$. Hence, $k=0$, which is a contradiction. Thus, $\Lambda_{1} \neq 0$ and we can apply Theorem 3 .

We have $h\left(\eta_{1}\right)=h(\delta)=\frac{1}{2} \log \delta$ and $h\left(\eta_{3}\right)=h(\alpha)=\frac{1}{3} \log \alpha$. Further,

$$
2 a=\frac{2 \alpha(\alpha+1)}{3 \alpha^{2}-1}
$$

the mimimal polynomial of $2 a$ is $23 x^{3}-46 x^{2}+24 x-8$ and has roots $2 a, 2 b, 2 c$. Since $2|b|=2|c|<1$ (by (11)), then

$$
h\left(\eta_{2}\right)=h(2 a)=\frac{1}{3}(\log 23+\log (2 a)) .
$$

On the other hand,

$$
\begin{aligned}
h\left(\eta_{4}\right) & =h\left(1+\alpha^{m-n}\right) \leq h(1)+h\left(\alpha^{m-n}\right)+\log 2 \\
& =(n-m) h(\alpha)+\log 2=\frac{1}{3}(n-m) \log \alpha+\log 2 .
\end{aligned}
$$

Thus, we can take $A_{1}:=3 \log \delta$,

$$
A_{2}:=2(\log 23+\log (2 a)), \quad A_{3}:=2 \log \alpha, \quad A_{4}:=2(n-m) \log \alpha+6 \log 2 .
$$

Now, Theorem 3 tells us that

$$
\begin{aligned}
\log \left|\Lambda_{1}\right|> & -1.4 \times 30^{7} \times 4^{4.5} \times 6^{2}(1+\log 6)(1+\log n)(3 \log \delta) \\
& \times(2(\log 23+\log (2 a))(2 \log \alpha)(2(n-m) \log \alpha+6 \log 2) \\
> & -2.33 \times 10^{17}(n-m)(\log n)(\log \delta) .
\end{aligned}
$$

Comparing the above inequality with 24 , we get

$$
n \log \alpha-\log 1.5<2.33 \times 10^{17}(n-m)(\log n)(\log \delta) .
$$

Hence, we get that

$$
n<8.30 \times 10^{17}(n-m)(\log n)(\log \delta) .
$$

We now return to the equation $x_{k}=P_{n}+P_{m}$ and rewrite it as

we obtain

$$
\frac{1}{2} \delta^{k}-a \alpha^{n}=-\frac{1}{2} \sigma^{k}+e(n)+P_{m},
$$

$$
\left|\delta^{k}(2 a)^{-1} \alpha^{-n}-1\right| \leq \frac{1}{a \alpha^{n-m}}\left(\frac{1}{\alpha}+\frac{1}{\alpha^{m+n / 2}}+\frac{1}{2 \delta^{k} \alpha^{m}}\right)<\frac{2.5}{\alpha^{n-m}} .
$$

Put

$$
\Lambda_{2}:=\delta^{k}(2 a)^{-1} \alpha^{-n}-1, \quad \Gamma_{2}:=k \log \delta-\log (2 a)-n \log \alpha .
$$

We assume for technical reasons that $n-m \geq 10$. So $\left|e^{\Lambda_{2}}-1\right|<\frac{1}{2}$. It follows that

$$
|k \log \delta-\log (2 a)-n \log \alpha|=\left|\Gamma_{2}\right|<e^{\left|\Lambda_{2}\right|}\left|e^{\Lambda_{2}}-1\right|<\frac{5}{\alpha^{n-m}} .
$$

Furthermore, $\Lambda_{2} \neq 0$ (so $\Gamma_{2} \neq 0$ ), since $\delta^{k} \in \mathbb{Q}(\alpha)$ by the previous argument.

We now apply Theorem 3 to the left-hand side of (27) with the data

$$
t:=3, \quad \eta_{1}:=\delta, \quad \eta_{2}:=2 a, \quad \eta_{3}:=\alpha, \quad b_{1}:=k, \quad b_{2}:=-1, \quad b_{3}:=-n .
$$

Thus, we have the same $A_{1}, A_{2}, A_{3}$ as before. Then, by Theorem 3 , we conclude that

$$
\log |\Lambda|>-9.82 \times 10^{14}(\log \delta)(\log n)(\log \alpha)
$$


By comparing with (27), we get

$$
n-m<9.84 \times 10^{14}(\log \delta)(\log n) .
$$

This was obtained under the assumption that $n-m \geq 10$, but if $n-m<10$, then the inequality also holds as well. We replace the bound (29) on $n-m$ in (26) and use the fact that $\delta^{k} \leq 2 \alpha^{n+3}$, to obtain bounds on $n$ and $k$ in terms of $\log n$ and $\log \delta$. We now record what we have proved so far.

Lemma 5. Let $(k, n, m)$ be a solution to the equation $x_{k}=P_{n}+P_{m}$ with $3 \leq$ $m<n$, then

$$
k<2.5 \times 10^{32}(\log n)^{2}(\log \delta) \quad \text { and } n<8.2 \times 10^{32}(\log n)^{2}(\log \delta)^{2} .
$$

4.2. Absolute bounds (I). We recall that $(k, n, m)=\left(k_{i}, n_{i}, m_{i}\right)$, where $3 \leq$ $m_{i}<n_{i}$, for $i=1,2$ and $1 \leq k_{1}<k_{2}$. Further, $n_{i} \geq 4$ for $i=1,2$. We return to (28) and write

$$
\left|\Gamma_{2}^{(i)}\right|:=\left|k_{i} \log \delta-\log (2 a)-n_{i} \log \alpha\right|<\frac{5}{\alpha^{n_{i}-m_{i}}}, \quad \text { for } \quad i=1,2 .
$$

We do a suitable cross product between $\Gamma_{2}^{(1)}, \Gamma_{2}^{(2)}$ and $k_{1}, k_{2}$ to eliminate the term involving $\log \delta$ in the above linear forms in logarithms:

$$
\begin{aligned}
\left|\Gamma_{3}\right| & :=\left|\left(k_{1}-k_{2}\right) \log (2 a)+\left(k_{1} n_{2}-k_{2} n_{1}\right) \log \alpha\right|=\left|k_{2} \Gamma_{2}^{(1)}-k_{1} \Gamma_{2}^{(2)}\right| \\
& \leq k_{2}\left|\Gamma_{2}^{(1)}\right|+k_{1}\left|\Gamma_{2}^{(2)}\right| \leq \frac{5 k_{2}}{\alpha^{n_{1}-m_{1}}}+\frac{5 k_{1}}{\alpha^{n_{2}-m_{2}}} \leq \frac{10 n_{2}}{\alpha^{\lambda}},
\end{aligned}
$$

where

$$
\lambda:=\min _{1 \leq i \leq 2}\left\{n_{i}-m_{i}\right\} .
$$

We need to find an upper bound for $\lambda$. If $10 n_{2} / \alpha^{\lambda}>1 / 2$, we then get

$$
\lambda<\frac{\log \left(20 n_{2}\right)}{\log \alpha}<4 \log \left(20 n_{2}\right) .
$$

Otherwise, $\left|\Gamma_{3}\right|<\frac{1}{2}$, so

$$
\left|e^{\Gamma_{3}}-1\right|=\left|(2 a)^{k_{1}-k_{2}} \alpha^{k_{1} n_{2}-k_{2} n_{1}}-1\right|<2\left|\Gamma_{3}\right|<\frac{20 n_{2}}{\alpha^{\lambda}} .
$$

We apply Theorem 3 with the data: $t:=2, \eta_{1}:=2 a, \eta_{2}:=\alpha, b_{1}:=k_{1}-k_{2}$, $b_{2}:=k_{1} n_{2}-k_{2} n_{1}$. We take the number field $\mathbb{K}:=\mathbb{Q}(\alpha)$ and $D=3$. We begin by checking that $e^{\Gamma_{3}}-1 \neq 0$ (so $\Gamma_{3} \neq 0$ ). This is true because $\alpha$ and $2 a$ are multiplicatively independent, since $\alpha$ is a unit in the ring of integers $\mathbb{Q}(\alpha)$ while the norm of $2 a$ is $8 / 23$.

We note that $\left|k_{1}-k_{2}\right|<k_{2}<n_{2}$. Further, from (31), we have

$$
\left|k_{2} n_{1}-k_{1} n_{2}\right|<\left(k_{2}-k_{1}\right) \frac{|\log (2 a)|}{\log \alpha}+\frac{10 k_{2}}{\alpha^{\lambda} \log \alpha}<11 k_{2}<11 n_{2}
$$

given that $\lambda \geq 1$. So, we can take $B:=11 n_{2}$. By Theorem 3 , with the same $A_{1}:=\log 23$ and $A_{2}:=\log \alpha$, we have that

$$
\log \left|e^{\Gamma_{3}}-1\right|>-1.55 \times 10^{11}\left(\log n_{2}\right)(\log \alpha) .
$$

By comparing this with (33), we get

$$
\lambda<1.56 \times 10^{11} \log n_{2} .
$$

Note that (34) is better than (32), so (34) always holds. Without loss of generality, we can assume that $\lambda=n_{i}-m_{i}$, for $i=1,2$ fixed.

We set $\{i, j\}=\{1,2\}$ and return to 25 to replace $(k, n, m)=\left(k_{i}, n_{i}, m_{i}\right)$ :

$$
\left|\Gamma_{1}^{(i)}\right|=\left|k_{i} \log \delta-\log (2 a)-n_{i} \log \alpha-\log \left(1+\alpha^{m_{i}-n_{i}}\right)\right|<\frac{3}{\alpha^{n_{i}}},
$$


and also return to (28), replacing with $(k, n, m)=\left(k_{j}, n_{j}, m_{j}\right)$ :

$$
\left|\Gamma_{2}^{(j)}\right|=\left|k_{j} \log \delta-\log (2 a)-n_{j} \log \alpha\right|<\frac{5}{\alpha^{n_{j}-m_{j}}} .
$$

We perform a cross product on (35) and (36) in order to eliminate the term on $\log \delta$ :

$$
\begin{aligned}
\left|\Gamma_{4}\right| & :=\left|\left(k_{j}-k_{i}\right) \log (2 a)+\left(k_{j} n_{i}-k_{i} n_{j}\right) \log \alpha+k_{j} \log \left(1+\alpha^{m_{i}-n_{i}}\right)\right| \\
& =\left|k_{i} \Gamma_{2}^{(j)}-k_{j} \Gamma_{1}^{(i)}\right| \leq k_{i}\left|\Gamma_{2}^{(j)}\right|+k_{j}\left|\Gamma_{1}^{(i)}\right| \\
& <\frac{5 k_{i}}{\alpha^{n_{j}-m_{j}}}+\frac{3 k_{j}}{\alpha^{n_{i}}}<\frac{8 n_{2}}{\alpha^{\nu}}
\end{aligned}
$$

with $\nu:=\min \left\{n_{i}, n_{j}-m_{j}\right\}$. As before, we need to find an upper bound on $\nu$. If $8 n_{2} / \alpha^{\nu}>1 / 2$, then we get

$$
\nu<\frac{\log \left(16 n_{2}\right)}{\log \alpha}<4 \log \left(16 n_{2}\right)
$$

Otherwise, $\left|\Gamma_{4}\right|<1 / 2$, so we have

$$
\left|e^{\Gamma_{4}}-1\right| \leq 2\left|\Gamma_{4}\right|<\frac{16 n_{2}}{\alpha^{\nu}} .
$$

In order to apply Theorem 3 , first if $e^{\Gamma_{4}}=1$, we obtain

$$
(2 a)^{k_{i}-k_{j}}=\alpha^{k_{j} n_{i}-k_{i} n_{j}}\left(1+\alpha^{-\lambda}\right)^{k_{j}} .
$$

Since $\alpha$ is a unit, the right-hand side in above is an algebraic integer. This is a contradiction because $k_{1}<k_{2}$ so $k_{i}-k_{j} \neq 0$, and neither $(2 a)$ nor $(2 a)^{-1}$ are algebraic intgers. Hence $e^{\Gamma_{4}} \neq 1$. By assuming that $\nu \geq 100$, we apply Theorem 3 with the data:

$$
\begin{gathered}
t:=3, \quad \eta_{1}:=2 a, \quad \eta_{2}:=\alpha, \quad \eta_{3}:=1+\alpha^{-\lambda}, \\
b_{1}:=k_{j}-k_{i}, \quad b_{2}:=k_{j} n_{i}-k_{i} n_{j}, \quad b_{3}:=k_{j},
\end{gathered}
$$

and the inequalities (34) and (39). We get

$$
\nu=\min \left\{n_{i}, n_{j}-m_{j}\right\}<1.14 \times 10^{14} \lambda \log n_{2}<1.78 \times 10^{25}\left(\log n_{2}\right)^{2} .
$$

The above inequality also holds when $\nu<100$. Further, it also holds when the inequality (38) holds. So the above inequality holds in all cases. Note that the case $\{i, j\}=\{2,1\}$ leads to $n_{1}-m_{1} \leq n_{1} \leq n_{2}+4$ whereas $\{i, j\}=\{1,2\}$ lead to $\nu=\min \left\{n_{1}, n_{2}-m_{2}\right\}$. Hence, either the minimum is $n_{1}$, so

$$
n_{1}<1.78 \times 10^{25}\left(\log n_{2}\right)^{2},
$$

or the minimum is $n_{j}-m_{j}$ and from the inequality (34) we get that

$$
\max _{1 \leq j \leq 2}\left\{n_{j}-m_{j}\right\}<1.78 \times 10^{25}\left(\log n_{2}\right)^{2} .
$$

Next, we assume that we are in the case (42). We evaluate 35 in $i=1,2$ and make a suitable cross product to eliminate the term involving $\log \delta$ :

$$
\begin{aligned}
\left|\Gamma_{5}\right|:= & \mid\left(k_{2}-k_{1}\right) \log (2 a)+\left(k_{2} n_{1}-k_{1} n_{2}\right) \log \alpha \\
& +k_{2} \log \left(1+\alpha^{m_{1}-n_{1}}\right)-k_{1} \log \left(1+\alpha^{m_{2}-n_{2}}\right) \mid \\
= & \left|k_{1} \Gamma_{1}^{(2)}-k_{2} \Gamma_{1}^{(1)}\right| \leq k_{1}\left|\Gamma_{1}^{(2)}\right|+k_{2}\left|\Gamma_{1}^{(1)}\right|<\frac{6 n_{2}}{\alpha^{n_{1}}} .
\end{aligned}
$$

In the above inequality we used the inequality 23 to conclude that $\min \left\{n_{1}, n_{2}\right\} \geq$ $n_{1}-4$ as well as the fact that $n_{i} \geq 4$ for $i=1.2$. Next, we apply a linear form in 
four logarithms to obtain an upper bound to $n_{1}$. As in the previous calculations, we pass from 43 to

$$
\left|e^{\Gamma_{5}}-1\right|<\frac{12 n_{2}}{\alpha^{n_{1}}}
$$

which is implied by 43 except if $n_{1}$ is very small, say

$$
n_{1} \leq 4 \log \left(12 n_{2}\right) .
$$

Thus, we assume that (45) does not hold, therefore (44) holds. Then to apply Theorem 3 , we first justify that $e^{\Gamma_{5}} \neq 1$. Otherwise,

$$
(2 a)^{k_{1}-k_{2}}=\alpha^{k_{2} n_{1}-k_{1} n_{2}}\left(1+\alpha^{n_{1}-m_{1}}\right)^{k_{2}}\left(1+\alpha^{n_{2}-m_{2}}\right)^{-k_{1}},
$$

By the fact that $k_{1}<k_{2}$, the norm $\mathbf{N}_{\mathbb{Q}(\alpha) / \mathbb{Q}}(2 a)=\frac{8}{23}$ and that $\alpha$ is a unit, we have that 23 divides the norm $\mathbf{N}_{\mathbb{K} / \mathbb{Q}}\left(1+\alpha^{n_{1}-m_{1}}\right)$. The factorization of the ideal generated by 23 in $\mathcal{O}_{\mathbb{Q}(\alpha)}$ is $(23)=\mathfrak{p}_{1}^{2} \mathfrak{p}_{2}$, where $\mathfrak{p}_{1}=(23, \alpha+13)$ and $\mathfrak{p}_{2}=(23, \alpha+20)$. Hence $\mathfrak{p}_{2}$ divides $\alpha^{n_{1}-m_{1}}+1$. Given that $\alpha \equiv-20\left(\bmod \mathfrak{p}_{2}\right)$, then $(-20)^{n_{1}-m_{1}} \equiv-1\left(\bmod \mathfrak{p}_{2}\right)$. Taking the norm $\mathbf{N}_{\mathbb{Q}(\alpha) / \mathbb{Q}}$, we obtain that $(-20)^{n_{1}-m_{1}} \equiv-1(\bmod 23)$. If $n_{1}-m_{1}$ is even -1 is a quadratic residue modulo 23 and if $n_{1}-m_{1}$ is odd then 20 is a quadratic residue modulo 23. But, neither -1 nor 20 are quadratic residues modulo 23 . Thus, $e^{\Gamma_{5}} \neq 1$.

Then, we apply Theorem 3 on the left-hand side of the inequalities (44) with the data

$$
\begin{gathered}
t:=4, \quad \eta_{1}:=2 a, \quad \eta_{2}:=\alpha, \quad \eta_{3}:=1+\alpha^{m_{1}-n_{1}}, \quad \eta_{4}:=1+\alpha^{m_{2}-n_{2}}, \\
b_{1}:=k_{2}-k_{1}, \quad b_{2}:=k_{2} n_{1}-k_{1} n_{2}, \quad b_{3}:=k_{2}, \quad b_{4}:=k_{1} .
\end{gathered}
$$

Together with combining the right-hand side of (44) with the inequalities (34) and 42, Theorem 3 gives

$$
\begin{aligned}
n_{1} & <3.02 \times 10^{16}\left(n_{1}-m_{1}\right)\left(n_{2}-m_{2}\right)\left(\log n_{2}\right) \\
& <8.33 \times 10^{52}\left(\log n_{2}\right)^{4} .
\end{aligned}
$$

In the above we used the facts that

$\min _{1 \leq i \leq 2}\left\{n_{i}-m_{i}\right\}<1.56 \times 10^{11} \log n_{2}$ and $\max _{1 \leq i \leq 2}\left\{n_{i}-m_{i}\right\}<1.78 \times 10^{25}\left(\log n_{2}\right)^{2}$.

This was obtained under the assumption that the inequality (45) does not hold. If (45) holds, then so does (46). Thus, we have that inequality (46) holds provided that inequality (42) holds. Otherwise, inequality (41) holds which is a better bound than (46). Hence, conclude that (46) holds in all posibble cases.

By the inequality $(22)$,

$$
\log \delta \leq k_{1} \log \delta \leq n_{1} \log \alpha+\log 6<2.38 \times 10^{52}\left(\log n_{2}\right)^{4} .
$$

By substituting this into 30 we get $n_{2}<4.64 \times 10^{137}\left(\log n_{2}\right)^{10}$, and then, by Lemma 4, with the data $r:=10, H:=4.64 \times 10^{137}$ and $L:=n_{2}$, we get that $n_{2}<4.87 \times 10^{165}$. This immediately gives that $n_{1}<1.76 \times 10^{63}$.

We record what we have proved.

Lemma 6. Let $\left(k_{i}, n_{i}, m_{i}\right)$ be a solution to $x_{k_{i}}=P_{n_{i}}+P_{m_{i}}$, with $3 \leq m_{i}<n_{i}$ for $i \in\{1,2\}$ and $1 \leq k_{1}<k_{2}$, then

$$
\max \left\{k_{1}, m_{1}\right\}<n_{1}<1.76 \times 10^{63} \text {, and } \max \left\{k_{2}, m_{2}\right\}<n_{2}<4.87 \times 10^{165} \text {. }
$$

\section{REDUCING THE BOUNDS FOR $n_{1}$ AND $n_{2}(\mathrm{I})$}

In this section we reduce the bounds for $n_{1}$ and $n_{2}$ given in Lemma 6 to cases that can be computationally treated. For this, we return to the inequalities for $\Gamma_{3}, \Gamma_{4}$ and $\Gamma_{5}$. 
5.1. The first reduction (I). We divide through both sides of the inequality (31) by $\left(k_{2}-k_{1}\right) \log \alpha$. We get that

$$
\left|\frac{\log (2 a)}{\log \alpha}-\frac{k_{2} n_{1}-k_{1} n_{2}}{k_{2}-k_{1}}\right|<\frac{36 n_{2}}{\alpha^{\lambda}\left(k_{2}-k_{1}\right)} \quad \text { with } \quad \lambda:=\min _{1 \leq i \leq 2}\left\{n_{i}-m_{i}\right\} .
$$

We assume that $\lambda \geq 10$. Below we apply Lemma 1 . We put $\tau:=\frac{\log (2 a)}{\log \alpha}$, which is irrational and compute its continued fraction

$$
\left[a_{0}, a_{1}, a_{2}, \ldots\right]=[1,3,3,1,11,1,2,1,1,1,3,1,1,1,2,5,1,15,2,19,1,1,2,2, \ldots]
$$

and its convergents

$$
\left[\frac{p_{0}}{q_{0}}, \frac{p_{1}}{q_{1}}, \frac{p_{2}}{q_{2}}, \ldots\right]=\left[1, \frac{4}{3}, \frac{13}{10}, \frac{17}{13}, \frac{200}{153}, \frac{217}{166}, \frac{634}{485}, \frac{851}{651}, \frac{1485}{1136}, \frac{2336}{1787}, \frac{8493}{6497}, \ldots\right] .
$$

Furthermore, we note that taking $M:=4.87 \times 10^{165}$ (by Lemma 6), it follows that

$q_{315}>M>n_{2}>k_{2}-k_{1} \quad$ and $\quad a(M):=\max \left\{a_{i}: 0 \leq i \leq 315\right\}=a_{282}=2107$.

Thus, by Lemma 1, we have that

$$
\left|\tau-\frac{k_{2} n_{1}-k_{1} n_{2}}{k_{2}-k_{1}}\right|>\frac{1}{2109\left(k_{2}-k_{1}\right)^{2}} .
$$

Hence, combining the inequalities (47) and (48), we obtain

$$
\alpha^{\lambda}<75924 n_{2}\left(k_{2}-k_{1}\right)<1.75 \times 10^{336},
$$

so $\lambda \leq 2714$. This was obtained under the assumption that $\lambda \geq 10$, Otherwise, $\lambda<10<2714$ holds as well.

Now, for each $n_{i}-m_{i}=\lambda \in[1,2714]$ we estimate a lower bound $\left|\Gamma_{4}\right|$, with

$$
\Gamma_{4}=\left(k_{j}-k_{i}\right) \log (2 a)+\left(k_{j} n_{i}-k_{i} n_{j}\right) \log \alpha+k_{j} \log \left(1+\alpha^{m_{i}-n_{i}}\right)
$$

given in the inequality 37, via the procedure described in Subsection 3.3 (LLLalgorithm). We recall that $\Gamma_{4} \neq 0$.

We apply Lemma 3 with the data:

$$
\begin{gathered}
t:=3, \quad \tau_{1}:=\log (2 a), \quad \tau_{2}:=\log \alpha, \quad \tau_{3}:=\log \left(1+\alpha^{-\lambda}\right), \\
x_{1}:=k_{j}-k_{i}, \quad x_{2}:=k_{j} n_{i}-k_{i} n_{j}, \quad x_{3}:=k_{j} .
\end{gathered}
$$

We set $X:=5.4 \times 10^{166}$ as an upper bound to $\left|x_{i}\right|<11 n_{2}$ for all $i=1,2,3$, and $C:=(20 X)^{5}$. A computer in Mathematica search allows us to conclude, together with the inequality (37), that

$$
2 \times 10^{-671}<\min _{1 \leq \lambda \leq 2714}\left|\Gamma_{4}\right|<8 n_{2} \alpha^{-\nu}, \quad \text { with } \quad \nu:=\min \left\{n_{i}, n_{j}-m_{j}\right\}
$$

which leads to $\nu \leq 6760$. As we have noted before, $\nu=n_{1}$ (so $n_{1} \leq 6760$ ) or $\nu=n_{j}-m_{j}$.

Next, we suppose that $n_{j}-m_{j}=\nu \leq 6760$. Since $\lambda \leq 2714$, we have

$$
\lambda:=\min _{1 \leq i \leq 2}\left\{n_{i}-m_{i}\right\} \leq 2714 \text { and } \chi:=\max _{1 \leq i \leq 2}\left\{n_{i}-m_{i}\right\} \leq 6760 .
$$

Now, returning to the inequality 43 which involves

$$
\begin{aligned}
\Gamma_{5}:= & \left(k_{2}-k_{1}\right) \log (2 a)+\left(k_{2} n_{1}-k_{1} n_{2}\right) \log \alpha \\
& +k_{2} \log \left(1+\alpha^{m_{1}-n_{1}}\right)-k_{1} \log \left(1+\alpha^{m_{2}-n_{2}}\right) \neq 0,
\end{aligned}
$$

we use again the LLL-algorithm to estimate the lower bound for $\left|\Gamma_{5}\right|$ and thus, find a bound for $n_{1}$ that is better than the one given in Lemma 6 .

We distinguish the cases $\lambda<\chi$ and $\lambda=\chi$. 
5.2. The case $\lambda<\chi$. We take $\lambda \in[1,2714]$ and $\chi \in[\lambda+1,6760]$ and apply Lemma 3 with the data: $t:=4$,

$$
\begin{gathered}
\tau_{1}:=\log (2 a), \quad \tau_{2}:=\log \alpha, \quad \tau_{3}:=\log \left(1+\alpha^{m_{1}-n_{1}}\right), \quad \tau_{4}:=\log \left(1+\alpha^{m_{2}-n_{2}}\right), \\
x_{1}:=k_{2}-k_{1}, \quad x_{2}:=k_{2} n_{1}-k_{1} n_{2}, \quad x_{3}:=k_{2}, \quad x_{4}:=-k_{1} .
\end{gathered}
$$

We also put $X:=5.4 \times 10^{166}$ and $C:=(20 X)^{9}$. After a computer search in Mathematica together with the inequality 43 , we can confirm that

$$
8 \times 10^{-1342}<\min _{\substack{1 \leq \lambda \leq 2714 \\ \lambda+1 \leq \chi \leq 6760}}\left|\Gamma_{5}\right|<6 n_{2} \alpha^{-n_{1}} .
$$

This leads to the inequality

$$
\alpha^{n_{1}}<7.5 \times 10^{1341} n_{2} .
$$

Subsitituting for the bound $n_{2}$ given in Lemma 6, we get that $n_{1} \leq 12172$.

5.3. The case $\lambda=\chi$. In this case, we have

$$
\Lambda_{5}:=\left(k_{2}-k_{1}\right)\left(\log (2 a)+\log \left(1+\alpha^{m_{1}-n_{1}}\right)\right)+\left(k_{2} n_{1}-k_{1} n_{2}\right) \log \alpha \neq 0 .
$$

We divide through the inequality 43 by $\left(k_{2}-k_{1}\right) \log \alpha$ to obtain

$$
\left|\frac{\log (2 a)+\log \left(1+\alpha^{m_{1}-n_{1}}\right)}{\log \alpha}-\frac{k_{2} n_{1}-k_{1} n_{2}}{k_{2}-k_{1}}\right|<\frac{21 n_{2}}{\alpha^{n_{1}}\left(k_{2}-k_{1}\right)}
$$

We now put

$$
\tau_{\lambda}:=\frac{\log (2 a)+\log \left(1+\alpha^{-\lambda}\right)}{\log \alpha}
$$

and compute its continued fractions $\left[a_{0}^{(\lambda)}, a_{1}^{(\lambda)}, a_{2}^{(\lambda)}, \ldots\right]$ and its convergents $\left[p_{0}^{(\lambda)} / q_{0}^{(\lambda)}, p_{1}^{(\lambda)} / q_{1}^{(\lambda)}, p_{2}^{(\lambda)} / q_{2}^{(\lambda)}, \ldots\right]$ for each $\lambda \in[1,2714]$. Furthermore, for each case we find an integer $t_{\lambda}$ such that $q_{t_{\lambda}}^{(\lambda)}>M:=4.87 \times 10^{165}>n_{2}>k_{2}-k_{1}$ and calculate

$$
a(M):=\max _{1 \leq \lambda \leq 2714}\left\{a_{i}^{(\lambda)}: 0 \leq i \leq t_{\lambda}\right\} .
$$

A computer search in Mathematica reveals that for $\lambda=321, t_{\lambda}=330$ and $i=263$, we have that $a(M)=a_{321}^{(330)}=306269$. Hence, combining the conclusion of Lemma 1 and the inequality (53), we get

$$
\alpha^{n_{1}}<21 \times 306271 n_{2}\left(k_{2}-k_{1}\right)<1.525 \times 10^{338},
$$

so $n_{1} \leq 2730$. Hence, we obtain that $n_{1} \leq 12172$ holds in all cases $\left(\nu=n_{1}, \lambda<\chi\right.$ or $\lambda=\chi)$. By the inequality $(22)$, we have that

$$
\log \delta \leq k_{1} \log \delta \leq n_{1} \log \alpha+\log 6<3475 .
$$

By considering the second inequality in (30), we can conclude that $n_{2} \leq 9.9 \times$ $10^{39}\left(\log n_{2}\right)^{2}$, which immediately yields $n_{2}<3.36 \times 10^{44}$, by a simple application of Lemma 4. We summarise the first cycle of our reduction process as follows:

$$
n_{1} \leq 12172 \text { and } n_{2} \leq 3.36 \times 10^{44} .
$$

From the above, we note that the upper bound on $n_{2}$ represents a very good reduction of the bound given in Lemma 6. Hence, we expect that if we restart our reduction cycle with the new bound on $n_{2}$, then we get a better bound on $n_{1}$. Thus, we return to the inequality (47) and take $M:=3.36 \times 10^{44}$. A computer search in Mathematica reveals that

$q_{88}>M>n_{2}>k_{2}-k_{1} \quad$ and $\quad a(M):=\max \left\{a_{i}: 0 \leq i \leq 88\right\}=a_{54}=373$, 
from which it follows that $\lambda \leq 752$. We now return to 49 and we put $X:=$ $3.36 \times 10^{44}$ and $C:=(10 X)^{5}$ and then apply the LLL-algorithm in Lemma 3 to $\lambda \in[1,752]$. After a computer search, we get

$$
5.33 \times 10^{-184}<\min _{1 \leq \lambda \leq 752}\left|\Gamma_{4}\right|<8 n_{2} \alpha^{-\nu}
$$

then $\nu \leq 1846$. By continuing under the assumption that $n_{j}-m_{j}=\nu \leq 1846$, we return to 50 and put $X:=3.36 \times 10^{44}, C:=(10 X)^{9}$ and $M:=3.36 \times 10^{44}$ for the case $\lambda<\chi$ and $\lambda=\chi$. After a computer search, we confirm that

$$
2 \times 10^{-366}<\min _{\substack{1 \leq \lambda \leq 752 \\ \lambda+1 \leq \chi \leq 1846}}\left|\Gamma_{5}\right|<6 n_{2} \alpha^{-n_{1}}
$$

gives $n_{1} \leq 3318$, and $a(M)=a_{175}^{(205)}=206961$, leads to $n_{1} \leq 772$. Hence, in both cases $n_{1} \leq 3318$ holds. This gives $n_{2} \leq 5 \times 10^{42}$ by a similar procedure as before, and $k_{1} \leq$.

We record what we have proved.

Lemma 7. Let $\left(k_{i}, n_{i}, m_{i}\right)$ be a solution to $X_{i}=P_{n_{i}}+P_{m_{i}}$, with $3 \leq m_{i}<n_{i}$ for $i=1,2$ and $1 \leq k_{1}<k_{2}$, then

$$
m_{1}<n_{1} \leq 3318, \quad k_{1} \leq 3125 \quad \text { and } \quad n_{2} \leq 5 \times 10^{42} .
$$

5.4. The final reduction (I). Returning back to (17) and $(19)$ and using the fact that $\left(x_{1}, y_{1}\right)$ is the smallest positive solution to the Pell equation (1), we obtain

$$
\begin{aligned}
x_{k} & =\frac{1}{2}\left(\delta^{k}+\sigma^{k}\right)=\frac{1}{2}\left(\left(x_{1}+y_{1} \sqrt{d}\right)^{k}+\left(x_{1}-y_{1} \sqrt{d}\right)^{k}\right) \\
& =\frac{1}{2}\left(\left(x_{1}+\sqrt{x_{1}^{2} \mp 1}\right)^{k}+\left(x_{1}-\sqrt{x_{1}^{2} \mp 1}\right)^{k}\right):=Q_{k}^{ \pm}\left(x_{1}\right) .
\end{aligned}
$$

Thus, we return to the Diophantine equation $x_{k_{1}}=P_{n_{1}}+P_{m_{1}}$ and consider the equations

$$
Q_{k_{1}}^{+}\left(x_{1}\right)=P_{n_{1}}+P_{m_{1}} \quad \text { and } \quad Q_{k_{1}}^{-}\left(x_{1}\right)=P_{n_{1}}+P_{m_{1}}
$$

with $k_{1} \in[1,3125], m_{1} \in[3,3318]$ and $n_{1} \in\left[m_{1}+1,3318\right]$.

Besides the trivial case $k_{1}=1$, with the help of a computer search in Mathematica on the above equations in (55), we list the only nontrivial solutions in Table 1. We also note that $3+2 \sqrt{2}=(1+\sqrt{2})^{2}$, so these solutions come from the same Pell equation when $d=2$. 


\begin{tabular}{|ccccc|}
\hline \multicolumn{5}{|c|}{$Q_{k_{1}}^{+}\left(x_{1}\right)$} \\
\hline$k_{1}$ & $x_{1}$ & $y_{1}$ & $d$ & $\delta$ \\
\hline 2 & 2 & 1 & 3 & $2+\sqrt{3}$ \\
2 & 3 & 2 & 2 & $3+2 \sqrt{2}$ \\
2 & 4 & 1 & 15 & $4+\sqrt{15}$ \\
2 & 5 & 2 & 6 & $5+2 \sqrt{6}$ \\
2 & 21 & 2 & 110 & $21+2 \sqrt{110}$ \\
2 & 22 & 1 & 483 & $22+\sqrt{483}$ \\
2 & 47 & 4 & 138 & $47+4 \sqrt{138}$ \\
\hline
\end{tabular}

\begin{tabular}{|ccccc|}
\hline \multicolumn{5}{|c|}{$Q_{k_{1}}^{-}\left(x_{1}\right)$} \\
\hline$k_{1}$ & $x_{1}$ & $y_{1}$ & $d$ & $\delta$ \\
\hline 2 & 1 & 1 & 2 & $1+\sqrt{2}$ \\
2 & 2 & 1 & 5 & $2+\sqrt{5}$ \\
2 & 3 & 1 & 10 & $3+\sqrt{10}$ \\
2 & 4 & 1 & 17 & $4+\sqrt{17}$ \\
2 & 5 & 1 & 26 & $5+\sqrt{26}$ \\
2 & 9 & 1 & 82 & $9+\sqrt{82}$ \\
2 & 10 & 1 & 101 & $10+\sqrt{101}$ \\
2 & 17 & 1 & 290 & $17+\sqrt{290}$ \\
2 & 42 & 1 & 1765 & $42+\sqrt{1765}$ \\
2 & 47 & 1 & 2210 & $47+\sqrt{2210}$ \\
2 & 63 & 1 & 3970 & $63+\sqrt{3970}$ \\
\hline
\end{tabular}

TABLE 1. Solutions to $Q_{k_{1}}^{ \pm}\left(x_{1}\right)=P_{n_{1}}+P_{m_{1}}$

From the above tables, we set each $\delta:=\delta_{t}$ for $t=1,2, \ldots 17$. We then work on the linear forms in logarithms $\Gamma_{1}$ and $\Gamma_{2}$, in order to reduce the bound on $n_{2}$ given in Lemma 7. From the inequality (28), for $(k, n, m):=\left(k_{2}, n_{2}, m_{2}\right)$, we write

$$
\left|k_{2} \frac{\log \delta_{t}}{\log \alpha}-n_{2}+\frac{\log (2 a)}{\log \left(\alpha^{-1}\right)}\right|<\left(\frac{5}{\log \alpha}\right) \alpha^{-\left(n_{2}-m_{2}\right)}
$$

for $t=1,2, \ldots 17$.

We put

$$
\tau_{t}:=\frac{\log \delta_{t}}{\log \alpha}, \quad \mu_{t}:=\frac{\log (2 a)}{\log \left(\alpha^{-1}\right)} \quad \text { and } \quad\left(A_{t}, B_{t}\right):=\left(\frac{5}{\log \alpha}, \alpha\right)
$$

We note that $\tau_{t}$ is transcendental by the Gelfond-Schneider's Theorem and thus, $\tau_{t}$ is irrational. We can rewrite the above inequality, 56 as

$$
0<\left|k_{2} \tau_{t}-n_{2}+\mu_{t}\right|<A_{t} B_{t}^{-\left(n_{2}-m_{2}\right)}, \quad \text { for } \quad t=1,2, \ldots, 17
$$

We take $M:=5 \times 10^{42}$ which is the upper bound on $n_{2}$ according to Lemma 7 and apply Lemma 2 to the inequality (57). As before, for each $\tau_{t}$ with $t=$ $1,2, \ldots, 17$, we compute its continued fraction $\left[a_{0}^{(t)}, a_{1}^{(t)}, a_{2}^{(t)}, \ldots\right]$ and its convergents $p_{0}^{(t)} / q_{0}^{(t)}, p_{1}^{(t)} / q_{1}^{(t)}, p_{2}^{(t)} / q_{2}^{(t)}, \ldots$ For each case, by means of a computer search in Mathematica, we find and integer $s_{t}$ such that

$$
q_{s_{t}}^{(t)}>3 \times 10^{43}=6 M \quad \text { and } \quad \epsilon_{t}:=|| \mu_{t} q^{(t)}\|-M\| \tau_{t} q^{(t)} \mid>0
$$

We finally compute all the values of $b_{t}:=\left\lfloor\log \left(A_{t} q_{s t}^{(t)} / \epsilon_{t}\right) / \log B_{t}\right\rfloor$. The values of $b_{t}$ correspond to the upper bounds on $n_{2}-m_{2}$, for each $t=1,2, \ldots, 17$, according to Lemma 2. The results of the computation for each $t$ are recorded in Table 2 . 


\begin{tabular}{llcccc}
\hline$t$ & $\delta_{t}$ & $s_{t}$ & $q_{s t}$ & $\epsilon_{t}>$ & $b_{t}$ \\
\hline 1 & $2+\sqrt{3}$ & 85 & $8.93366 \times 10^{43}$ & 0.3100 & 374 \\
2 & $4+\sqrt{15}$ & 90 & $3.90052 \times 10^{43}$ & 0.3124 & 371 \\
3 & $5+2 \sqrt{6}$ & 80 & $3.16032 \times 10^{43}$ & 0.0122 & 382 \\
4 & $21+2 \sqrt{110}$ & 88 & $6.33080 \times 10^{43}$ & 0.2200 & 374 \\
5 & $22+\sqrt{483}$ & 75 & $4.19689 \times 10^{43}$ & 0.2361 & 372 \\
6 & $47+4 \sqrt{138}$ & 96 & $7.76442 \times 10^{43}$ & 0.3732 & 373 \\
7 & $1+\sqrt{2}$ & 78 & $1.46195 \times 10^{44}$ & 0.3328 & 375 \\
8 & $2+\sqrt{5}$ & 94 & $1.48837 \times 10^{44}$ & 0.2146 & 377 \\
9 & $3+\sqrt{10}$ & 88 & $4.21425 \times 10^{43}$ & 0.1347 & 374 \\
10 & $4+\sqrt{17}$ & 92 & $1.11753 \times 10^{44}$ & 0.2529 & 375 \\
11 & $5+\sqrt{26}$ & 98 & $3.23107 \times 10^{43}$ & 0.1043 & 374 \\
12 & $9+\sqrt{82}$ & 74 & $5.25207 \times 10^{43}$ & 0.2181 & 373 \\
13 & $10+\sqrt{101}$ & 94 & $1.86122 \times 10^{44}$ & 0.2672 & 377 \\
14 & $17+\sqrt{290}$ & 87 & $1.06422 \times 10^{44}$ & 0.0193 & 384 \\
15 & $42+\sqrt{1765}$ & 78 & $3.81406 \times 10^{43}$ & 0.1768 & 373 \\
16 & $47+\sqrt{2210}$ & 94 & $3.92482 \times 10^{43}$ & 0.4476 & 370 \\
17 & $63+\sqrt{3970}$ & 85 & $6.00550 \times 10^{43}$ & 0.4056 & 371 \\
\hline
\end{tabular}

TABLE 2. First reduction computation results (I)

By replacing $(k, n, m):=\left(k_{2}, n_{2}, m_{2}\right)$ in the inequality (25), we can write

$$
\left|k_{2} \frac{\log \delta_{t}}{\log \alpha}-n_{2}+\frac{\log \left(2 a\left(1+\alpha^{-\left(n_{2}-m_{2}\right)}\right)\right)}{\log \left(\alpha^{-1}\right)}\right|<\left(\frac{3}{\log \alpha}\right) \alpha^{-n_{2}},
$$

for $t=1,2, \ldots, 17$.

We now put

$\tau_{t}:=\frac{\log \delta_{t}}{\log \alpha}, \quad \mu_{t, n_{2}-m_{2}}:=\frac{\log \left(2 a\left(1+\alpha^{-\left(n_{2}-m_{2}\right)}\right)\right)}{\log \left(\alpha^{-1}\right)} \quad$ and $\quad\left(A_{t}, B_{t}\right):=\left(\frac{3}{\log \alpha}, \alpha\right)$.

With the above notations, we can rewrite $(58)$ as

$$
0<\left|k_{2} \tau_{t}-n_{2}+\mu_{t, n_{2}-m_{2}}\right|<A_{t} B_{t}^{-n_{2}}, \quad \text { for } \quad t=1,2, \ldots 17 .
$$

We again apply Lemma 2 to the above inequality (59), for

$$
t=1,2, \ldots, 17, \quad n_{2}-m_{2}=1,2, \ldots, b_{t}, \quad \text { with } \quad M:=5 \times 10^{43} .
$$

We take

$$
\epsilon_{t, n_{2}-m_{2}}:=\left\|\mu_{t} q^{\left(t, n_{2}-m_{2}\right)}\right\|-M\left\|\tau_{t} q^{\left(t, n_{2}-m_{2}\right)}\right\|>0
$$

and

$$
b_{t}=b_{t, n_{2}-m_{2}}:=\left\lfloor\log \left(A_{t} q_{s t}^{\left(t, n_{2}-m_{2}\right)} / \epsilon_{t, n_{2}-m_{2}}\right) / \log B_{t}\right\rfloor .
$$

With the help of Mathematica, we obtain the results in Table 3 .

\begin{tabular}{c|ccccccccc}
\hline$t$ & 1 & 2 & 3 & 4 & 5 & 6 & 7 & 8 & 9 \\
$b_{t, n_{2}-m_{2}}$ & 388 & 389 & 394 & 394 & 393 & 394 & 396 & 392 & 392 \\
\hline$t$ & 10 & 11 & 12 & 13 & 14 & 15 & 16 & 17 & \\
$b_{t, n_{2}-m_{2}}$ & 396 & 392 & 408 & 390 & 396 & 396 & 388 & 389 & \\
\hline
\end{tabular}

TABLE 3. Final reduction computation results (I) 
Thus, $\max \left\{b_{t, n_{2}-m_{2}}: t=1,2, \ldots, 17\right.$ and $\left.n_{2}-m_{2}=1,2, \ldots b_{t}\right\} \leq 408$.

Thus, by Lemma 2, we have that $n_{2} \leq 408$, for all $t=1,2, \ldots, 17$, and by the inequality (23) we have that $n_{1} \leq n_{2}+4$. From the fact that $\delta^{k} \leq 2 \alpha^{n+3}$, we can conclude that $k_{1}<k_{2} \leq 133$. Collecting everything together, our problem is reduced to search for the solutions for (21) in the following range

$$
1 \leq k_{1}<k_{2} \leq 133, \quad 0 \leq m_{1}<n_{1} \in[3,408] \quad \text { and } \quad 0 \leq m_{2}<n_{2} \in[3,408] .
$$

After a computer search on the equation (21) on the above ranges, we obtained the following solutions, which are the only solutions for the exceptional $d$ cases we have stated in Theorem 1:

For the +1 case:

$$
\begin{array}{cl}
(d=2) & x_{1}=3=P_{6}+P_{0}=P_{5}+P_{3}, \quad x_{2}=17=P_{12}+P_{3} ; \\
(d=3) & x_{1}=2=P_{3}+P_{0}=P_{3}+P_{3}, x_{2}=7=P_{9}+P_{0}=P_{7}+P_{6}, \\
& x_{3}=26=P_{13}+P_{8} ; \\
(d=6) & x_{1}=5=P_{8}+P_{0}=P_{7}+P_{3}=P_{6}+P_{5}, \\
& x_{2}=49=P_{16}+P_{0}=P_{15}+P_{12}=P_{14}+P_{13} ; \\
(d=15) & x_{1}=4=P_{7}+P_{0}=P_{6}+P_{3}=P_{5}+P_{5}, \quad x_{2}=31=P_{14}+P_{6} ; \\
(d=110) & x_{1}=21=P_{13}+P_{0}=P_{12}+P_{8}=P_{11}+P_{10}, \\
& x_{2}=881=P_{26}+P_{17}=P_{25}+P_{22} ; \\
(d=483) & x_{1}=22=P_{13}+P_{3}, \quad x_{2}=967=P_{26}+P_{20}=P_{25}+P_{23} .
\end{array}
$$

For the -1 case:

$$
\begin{array}{ll}
(d=2) & x_{1}=1=P_{3}+P_{0}, \quad x_{2}=7=P_{9}+P_{0}=P_{8}+P_{5}=P_{7}+P_{6}, \\
& x_{3}=41=P_{15}+P_{7}=P_{14}+P_{10}=P_{13}+P_{12} \\
(d=5) & x_{1}=2=P_{5}+P_{0}=P_{3}+P_{3}, \quad x_{2}=38=P_{15}+P_{3} \\
(d=10) & x_{1}=3=P_{6}+P_{0}=P_{5}+P_{3}, \quad x_{2}=117=P_{19}+P_{6} \\
(d=17) & x_{1}=4=P_{7}+P_{0}=P_{6}+P_{3}=P_{5}+P_{5}, \quad x_{2}=P_{22}+P_{6} .
\end{array}
$$

This completes the proof of Theorem 1 .

\section{Proof of Theorem 2}

The proof of Theorem 2 will be similar to that of Theorem 1. We also give the details for the benefit of the reader. Further, for technical reasons in our proof, we assume that $d \geq 5$ and then treat the cases $d \in\{2,3\}$ during the reduction procedure.

Let $\left(X_{1}, Y_{1}\right)$ be the smallest positive integer solution to the Pell quation (2). We Put

$$
\rho:=\frac{X_{1}+Y_{1} \sqrt{d}}{2} \quad \text { and } \quad \varrho=\frac{X_{1}-Y_{1} \sqrt{d}}{2} .
$$

From which we get that

$$
\rho \cdot \varrho=\frac{X_{1}^{2}-d Y_{1}^{2}}{4}=: \epsilon, \quad \text { where } \quad \epsilon \in\{ \pm 1\} .
$$

Then

$$
X_{n}=\rho^{k}+\varrho^{k} .
$$


Since $\rho \geq \frac{1+\sqrt{5}}{2}$, it follows that the estimate

$$
\frac{\rho^{k}}{\alpha^{2}} \leq X_{k} \leq 2 \rho^{k} \quad \text { holds for all } \quad k \geq 1
$$

Similarly, as before, we assume that $\left(k_{1}, n_{1}, m_{1}\right)$ and $\left(k_{2}, n_{2}, m_{2}\right)$ are triples of integers such that

$$
X_{k_{1}}=P_{n_{1}}+P_{m_{1}} \quad \text { and } \quad X_{k_{2}}=P_{n_{2}}+P_{m_{2}}
$$

We asuume that $1 \leq k_{1}<k_{2}$. We also assume that $4 \leq m_{j}<n_{j}$ for $j=1,2$. We set $(k, n, m):=\left(k_{j}, n_{j}, m_{j}\right)$, for $j=1,2$. Using the inequalities (12) and (63), we get from (64) that

$$
\frac{\rho^{k}}{\alpha^{2}} \leq X_{k}=P_{n}+P_{m} \leq 2 \alpha^{n-1} \quad \text { and } \quad \alpha^{n-2} \leq P_{n}+P_{m}=X_{k} \leq 2 \rho^{k} .
$$

The above inequalities give

$$
(n-2) \log \alpha-\log 2<k \log \rho<(n+1) \log \alpha+\log 2 .
$$

Dividing through by $\log \alpha$ and setting $c_{1}:=1 / \log \alpha$, as before, we get that

$$
-2-c_{1} \log 2<c_{1} k \log \rho-n<1+c_{1} \log 2,
$$

and since $\alpha^{3}>2$, we get

$$
\left|n-c_{1} \log \rho\right|<5 .
$$

Furthermore, $k<n$, for if not, we would then get that

$$
\rho^{n} \leq \rho^{k}<2 \alpha^{n+1}, \quad \text { implying }\left(\frac{\rho}{\alpha}\right)^{n}<2 \alpha,
$$

which is false since $\rho \leq \frac{1+\sqrt{5}}{2}, 1.32<\alpha<1.33$ and $n \geq 5$.

Besides, given that $k_{1}<k_{2}$, we have by (13) and (64) that

$$
\alpha^{n_{1}-2} \leq P_{n_{1}} \leq P_{n_{1}}+P_{m_{1}}=X_{k_{1}}<X_{k_{2}}=P_{n_{2}}+P_{m_{2}} \leq 2 P_{n_{2}}<2 \alpha^{n_{2}-1} \text {. }
$$

Thus, as before, we get that

$$
n_{1}<n_{2}+4 \text {. }
$$

6.1. An inequality for $n$ and $k$ (II). Using the equations (9) and (60) and (64), we get

$$
\rho^{k}+\varrho^{k}=P_{n}+P_{m}=a \alpha^{n}+e(n)+a \alpha^{m}+e(m)
$$

So,

$$
\rho^{k}-a\left(\alpha^{n}+\alpha^{m}\right)=-\varrho^{k}+e(n)+e(m),
$$

and by 12 , we have

$$
\begin{aligned}
\left|\rho^{k} a^{-1} \alpha^{-n}\left(1+\alpha^{m-n}\right)^{-1}-1\right| \leq & \frac{1}{\rho^{k} a\left(\alpha^{n}+\alpha^{m}\right)}+\frac{2|b|}{\alpha^{n / 2} a\left(\alpha^{n}+\alpha^{m}\right)} \\
& +\frac{2|b|}{\alpha^{m / 2} a\left(\alpha^{n}+\alpha^{m}\right)} \\
\leq & \frac{1}{a \alpha^{n}}\left(\frac{1}{\rho^{k}}+\frac{2|b|}{\alpha^{n / 2}}+\frac{2|b|}{\alpha^{m / 2}}\right)<\frac{2.5}{\alpha^{n}} .
\end{aligned}
$$

Thus, we have

$$
\left|\rho^{k} a^{-1} \alpha^{-n}\left(1+\alpha^{m-n}\right)^{-1}-1\right|<\frac{2.5}{\alpha^{n}} .
$$

Put

$$
\Lambda_{1}^{\prime}:=\rho^{k} a^{-1} \alpha^{-n}\left(1+\alpha^{m-n}\right)^{-1}-1
$$


and

$$
\Gamma_{1}^{\prime}:=k \log \rho-\log a-n \log \alpha-\log \left(1+\alpha^{m-n}\right) .
$$

Since $\left|\Lambda_{1}^{\prime}\right|=\left|e^{\Gamma_{1}^{\prime}}-1\right|<0.83$ for $n \geq 4$ (because $2.5 / \alpha^{4}<0.83$ ), it follows that $e^{\left|\Gamma_{1}^{\prime}\right|}<4$ and so

$$
\left|\Gamma_{1}^{\prime}\right|<e^{\left|\Gamma_{1}^{\prime}\right|}\left|e^{\Gamma_{1}^{\prime}}-1\right|<\frac{10}{\alpha^{n}}
$$

Thus, we get that

$$
\left|k \log \rho-\log a-n \log \alpha-\log \left(1+\alpha^{m-n}\right)\right|<\frac{10}{\alpha^{n}} .
$$

We apply Theorem 3 on the left-hand side of (67) with the data:

$$
\begin{aligned}
t:=4, \quad \eta_{1}:=\rho, \quad \eta_{2}:=a, \quad \eta_{3}:=\alpha, \quad \eta_{4}:=1+\alpha^{m-n}, \\
b_{1}:=k, \quad b_{2}:=-1, \quad b_{3}:=-n, \quad b_{4}:=-1 .
\end{aligned}
$$

Furthermore, we take same the number field as before, $\mathbb{K}=\mathbb{Q}(\sqrt{d}, \alpha)$ with degree $D=6$. We also take $D_{\mathbb{K}}=n$. First we note that the left-hand side of (24) is non-zero, since otherwise,

$$
\rho^{k}=a\left(\alpha^{n}+\alpha^{m}\right) .
$$

By the same argument as before, we get a contradiction. Thus, $\Lambda_{1}^{\prime} \neq 0$ and we can apply Theorem 3. Further,

$$
a=\frac{\alpha(\alpha+1)}{3 \alpha^{2}-1}
$$

the mimimal polynomial of $a$ is $23 x^{3}-23 x^{2}+6 x-1$ and has roots $a, b, c$. Since $\max \{a, b, c\}<1$ (by (11)), then $h\left(\eta_{2}\right)=h(a)=\frac{1}{3} \log 23$. Thus, we can take $A_{1}:=3 \log \rho, A_{2}:=2 \log 23, A_{3}:=2 \log \alpha$, and $A_{4}:=2(n-m) \log \alpha+6 \log 2$.

Now, Theorem 3 tells us that

$$
\begin{aligned}
\log \left|\Lambda_{1}^{\prime}\right|> & -1.4 \times 30^{7} \times 4^{4.5} \times 6^{2}(1+\log 6)(1+\log n)(3 \log \rho) \\
& \times(2 \log 23)(2 \log \alpha)(2(n-m) \log \alpha+6 \log 2) \\
> & -2.08 \times 10^{17}(n-m)(\log n)(\log \rho) .
\end{aligned}
$$

Comparing the above inequality with (67), we get

$$
n \log \alpha-\log 2.5<2.08 \times 10^{17}(n-m)(\log n)(\log \rho) .
$$

Hence, we get that

$$
n<7.40 \times 10^{17}(n-m)(\log n)(\log \rho) .
$$

We now return to the equation $X_{k}=P_{n}+P_{m}$ and rewrite it as

$$
\rho^{k}-a \alpha^{n}=-\varrho^{k}+e(n)+P_{m},
$$

we obtain

$$
\left|\rho^{k} a^{-1} \alpha^{-n}-1\right| \leq \frac{1}{a \alpha^{n-m}}\left(\frac{1}{\alpha}+\frac{1}{\alpha^{m+n / 2}}+\frac{1}{\rho^{k} \alpha^{m}}\right)<\frac{3}{\alpha^{n-m}} .
$$

Put

$$
\Lambda_{2}^{\prime}:=\rho^{k} a^{-1} \alpha^{-n}-1, \quad \Gamma_{2}^{\prime}:=k \log \rho-\log a-n \log \alpha .
$$

We assume for technical reasons that $n-m \geq 10$. So $\left|e^{\Lambda_{2}}-1\right|<\frac{1}{2}$. It follows that

$$
|k \log \rho-\log a-n \log \alpha|=\left|\Gamma_{2}^{\prime}\right|<e^{\left|\Lambda_{2}^{\prime}\right|}\left|e^{\Lambda_{2}^{\prime}}-1\right|<\frac{6}{\alpha^{n-m}} .
$$

Furthermore, $\Lambda_{2}^{\prime} \neq 0$ (so $\Gamma_{2}^{\prime} \neq 0$ ), since $\rho^{k} \in \mathbb{Q}(\alpha)$ by the previous argument. 
We now apply Theorem 3 to the left-hand side of $(70)$ with the data $t:=3, \quad \eta_{1}:=\rho, \quad \eta_{2}:=a, \quad \eta_{3}:=\alpha, \quad b_{1}:=k, \quad b_{2}:=-1, \quad b_{3}:=-n$.

Thus, we have the same $A_{1}, A_{2}, A_{3}$ as before. Then, by Theorem 3 , we conclude that

$$
\log |\Lambda|>-9.50 \times 10^{14}(\log \rho)(\log n)(\log \alpha) .
$$

By comparing with 70 , we get

$$
n-m<9.52 \times 10^{14}(\log \rho)(\log n) .
$$

This was obtained under the assumption that $n-m \geq 10$, but if $n-m<10$, then the inequality also holds as well. We replace the bound $(72)$ on $n-m$ in (69) and use the fact that $\rho^{k} \leq 2 \alpha^{n+1}$, to obtain bounds on $n$ and $k$ in terms of $\log n$ and $\log \rho$. We again record what we have proved.

Lemma 8. Let $(k, n, m)$ be a solution to the equation $X_{k}=P_{n}+P_{m}$ with $3 \leq$ $m<n$, then

$$
k<1.98 \times 10^{32}(\log n)^{2}(\log \rho) \quad \text { and } \quad n<7.03 \times 10^{32}(\log n)^{2}(\log \rho)^{2} .
$$

6.2. Absolute bounds (II). We recall that $(k, n, m)=\left(k_{j}, n_{j}, m_{j}\right)$, where $3 \leq$ $m_{j}<n_{j}$, for $j=1,2$ and $1 \leq k_{1}<k_{2}$. Further, $n_{j} \geq 4$ for $j=1,2$. We return to (71) and write

$$
\left|\Gamma_{2}^{(j)^{\prime}}\right|:=\left|k_{j} \log \rho-\log a-n_{j} \log \alpha\right|<\frac{6}{\alpha^{n_{j}-m_{j}}}, \quad \text { for } \quad j=1,2 .
$$

We do a suitable cross product between $\Gamma_{2}^{(1)^{\prime}}, \Gamma_{2}^{(2)^{\prime}}$ and $k_{1}, k_{2}$ to eliminate the term involving $\log \rho$ in the above linear forms in logarithms:

$$
\begin{aligned}
\left|\Gamma_{3}^{\prime}\right| & :=\left|\left(k_{1}-k_{2}\right) \log a+\left(k_{1} n_{2}-k_{2} n_{1}\right) \log \alpha\right|=\left|k_{2} \Gamma_{2}^{(1)^{\prime}}-k_{1} \Gamma_{2}^{(2)^{\prime}}\right| \\
& \leq k_{2}\left|\Gamma_{2}^{(1)^{\prime}}\right|+k_{1}\left|\Gamma_{2}^{(2)^{\prime}}\right| \leq \frac{6 k_{2}}{\alpha^{n_{1}-m_{1}}}+\frac{6 k_{1}}{\alpha^{n_{2}-m_{2}}} \leq \frac{12 n_{2}}{\alpha^{\lambda^{\prime}}},
\end{aligned}
$$

where

$$
\lambda^{\prime}:=\min _{1 \leq j \leq 2}\left\{n_{j}-m_{j}\right\}
$$

We need to find an upper bound for $\lambda^{\prime}$. If $12 n_{2} / \alpha^{\lambda^{\prime}}>1 / 2$, we then get

$$
\lambda^{\prime}<\frac{\log \left(24 n_{2}\right)}{\log \alpha}<4 \log \left(24 n_{2}\right) .
$$

Otherwise, $\left|\Gamma_{3}^{\prime}\right|<\frac{1}{2}$, so

$$
\left|e^{\Gamma_{3}^{\prime}}-1\right|=\left|a^{k_{1}-k_{2}} \alpha^{k_{1} n_{2}-k_{2} n_{1}}-1\right|<2\left|\Gamma_{3}^{\prime}\right|<\frac{24 n_{2}}{\alpha^{\lambda^{\prime}}} .
$$

We apply Theorem 3 with the data: $t:=2, \eta_{1}:=a, \eta_{2}:=\alpha, b_{1}:=k_{1}-k_{2}$, $b_{2}:=k_{1} n_{2}-k_{2} n_{1}$. We take the number field $\mathbb{K}:=\mathbb{Q}(\alpha)$ and $D=3$. We begin by checking that $e^{\Gamma_{3}^{\prime}}-1 \neq 0\left(\right.$ so $\left.\Gamma_{3}^{\prime} \neq 0\right)$. This is true because $\alpha$ and $a$ are multiplicatively independent, since $\alpha$ is a unit in the ring of integers $\mathbb{Q}(\alpha)$ while the norm of $a$ is $1 / 23$.

We note that $\left|k_{1}-k_{2}\right|<k_{2}<n_{2}$. Further, from (74), we have

$$
\left|k_{2} n_{1}-k_{1} n_{2}\right|<\left(k_{2}-k_{1}\right) \frac{|\log a|}{\log \alpha}+\frac{12 k_{2}}{\alpha^{\lambda} \log \alpha}<13 k_{2}<13 n_{2}
$$

given that $\lambda \geq 1$. So, we can take $B:=13 n_{2}$. By Theorem 3 , with the same $A_{1}:=\log 23$ and $A_{2}:=\log \alpha$, we have that

$$
\log \left|e^{\Gamma_{3}^{\prime}}-1\right|>-4.63 \times 10^{10}\left(\log n_{2}\right)(\log \alpha) .
$$


By comparing this with $(76)$, we get

$$
\lambda^{\prime}<1.62 \times 10^{11} \log n_{2} .
$$

Note that $(77)$ is better than $(76)$, so $(77)$ always holds. Without loss of generality, we can assume that $\lambda^{\prime}=n_{j}-m_{j}$, for $j=1,2$ fixed.

We set $\{j, i\}=\{1,2\}$ and return to $(68)$ to replace $(k, n, m)=\left(k_{i}, n_{i}, m_{i}\right)$ :

$$
\left|\Gamma_{1}^{(i)^{\prime}}\right|=\left|k_{i} \log \rho-\log a-n_{i} \log \alpha-\log \left(1+\alpha^{m_{i}-n_{i}}\right)\right|<\frac{10}{\alpha^{n_{i}}},
$$

and also return to (71), with $(k, n, m)=\left(k_{j}, n_{j}, m_{j}\right)$ :

$$
\left|\Gamma_{2}^{(j)^{\prime}}\right|=\left|k_{j} \log \rho-\log a-n_{j} \log \alpha\right|<\frac{6}{\alpha^{n_{j}-m_{j}}} .
$$

We perform a cross product on $(78)$ and $(79)$ in order to eliminate the term on $\log \rho$ :

$$
\begin{aligned}
\left|\Gamma_{4}^{\prime}\right| & :=\left|\left(k_{j}-k_{i}\right) \log a+\left(k_{j} n_{i}-k_{i} n_{j}\right) \log \alpha+k_{j} \log \left(1+\alpha^{m_{i}-n_{i}}\right)\right| \\
& =\left|k_{i} \Gamma_{2}^{(j)^{\prime}}-k_{j} \Gamma_{1}^{(i)^{\prime}}\right| \leq k_{i}\left|\Gamma_{2}^{(j)^{\prime}}\right|+k_{j}\left|\Gamma_{1}^{(i)^{\prime}}\right| \\
& <\frac{6 k_{i}}{\alpha^{n_{j}-m_{j}}}+\frac{10 k_{j}}{\alpha^{n_{i}}}<\frac{16 n_{2}}{\alpha^{\nu^{\prime}}}
\end{aligned}
$$

with $\nu^{\prime}:=\min \left\{n_{i}, n_{j}-m_{j}\right\}$. As before, we need to find an upper bound on $\nu^{\prime}$. If $16 n_{2} / \alpha^{\nu^{\prime}}>1 / 2$, then we get

$$
\nu^{\prime}<\frac{\log \left(32 n_{2}\right)}{\log \alpha}<4 \log \left(32 n_{2}\right) .
$$

Otherwise, $\left|\Gamma_{4}^{\prime}\right|<1 / 2$, so we have

$$
\left|e^{\Gamma_{4}^{\prime}}-1\right| \leq 2\left|\Gamma_{4}^{\prime}\right|<\frac{32 n_{2}}{\alpha^{\nu^{\prime}}} .
$$

In order to apply Theorem 3 , first if $e^{\Gamma_{4}^{\prime}}=1$, we obtain

$$
a^{k_{i}-k_{j}}=\alpha^{k_{j} n_{i}-k_{i} n_{j}}\left(1+\alpha^{-\lambda^{\prime}}\right)^{k_{j}} .
$$

Since $\alpha$ is a unit, the right-hand side in above is an algebraic integer. This is a contradiction because $k_{1}<k_{2}$ so $k_{i}-k_{j} \neq 0$, and neither $a$ nor $a^{-1}$ are algebraic intgers. Hence $e^{\Gamma_{4}^{\prime}} \neq 1$. By assuming that $\nu^{\prime} \geq 100$, we apply Theorem 3 with the data:

$$
\begin{gathered}
t:=3, \quad \eta_{1}:=a, \quad \eta_{2}:=\alpha, \quad \eta_{3}:=1+\alpha^{-\lambda^{\prime}}, \\
b_{1}:=k_{j}-k_{i}, \quad b_{2}:=k_{j} n_{i}-k_{i} n_{j}, \quad b_{3}:=k_{j},
\end{gathered}
$$

and the inequalities (77) and (82). We get

$$
\nu^{\prime}=\min \left\{n_{i}, n_{j}-m_{j}\right\}<1.85 \times 10^{13} \lambda^{\prime} \log n_{2}<3 \times 10^{24}\left(\log n_{2}\right)^{2} .
$$

The above inequality also holds when $\nu^{\prime}<100$. Further, it also holds when the inequality (81) holds. So the above inequality holds in all cases. Note that the case $\{i, j\}=\{2,1\}$ leads to $n_{1}-m_{1} \leq n_{1} \leq n_{2}+4$ whereas $\{i, j\}=\{1,2\}$ lead to $\nu^{\prime}=\min \left\{n_{1}, n_{2}-m_{2}\right\}$. Hence, either the minimum is $n_{1}$, so

$$
n_{1}<3 \times 10^{24}\left(\log n_{2}\right)^{2},
$$

or the minimum is $n_{j}-m_{j}$ and from the inequality (34) we get that

$$
\max _{1 \leq j \leq 2}\left\{n_{j}-m_{j}\right\}<3 \times 10^{24}\left(\log n_{2}\right)^{2} .
$$


Next, we assume that we are in the case (85). We evaluate $(78)$ in $i=1,2$ and make a suitable cross product to eliminate the term involving $\log \rho$ :

$$
\begin{aligned}
\left|\Gamma_{5}^{\prime}\right|:= & \mid\left(k_{2}-k_{1}\right) \log a+\left(k_{2} n_{1}-k_{1} n_{2}\right) \log \alpha \\
& +k_{2} \log \left(1+\alpha^{m_{1}-n_{1}}\right)-k_{1} \log \left(1+\alpha^{m_{2}-n_{2}}\right) \mid \\
= & \left|k_{1} \Gamma_{1}^{(2)}-k_{2} \Gamma_{1}^{(1)}\right| \leq k_{1}\left|\Gamma_{1}^{(2)}\right|+k_{2}\left|\Gamma_{1}^{(1)}\right|<\frac{20 n_{2}}{\alpha^{n_{1}}} .
\end{aligned}
$$

In the above inequality we used the inequality 66 to conclude that $\min \left\{n_{1}, n_{2}\right\} \geq$ $n_{1}-4$ as well as the fact that $n_{i} \geq 4$ for $i=1.2$. Next, we apply a linear form in four logarithms to obtain an upper bound to $n_{1}$. As in the previous calculations, we pass from (86) to

$$
\left|e^{\Gamma_{5}^{\prime}}-1\right|<\frac{40 n_{2}}{\alpha^{n_{1}}}
$$

which is implied by 86 except if $n_{1}$ is very small, say

$$
n_{1} \leq 4 \log \left(40 n_{2}\right) .
$$

Thus, we assume that 88$)$ does not hold, therefore $(87)$. Then to apply Theorem 3 . we first justify that $e^{\Gamma_{5}^{\prime}} \neq 1$. Otherwise,

$$
a^{k_{1}-k_{2}}=\alpha^{k_{2} n_{1}-k_{1} n_{2}}\left(1+\alpha^{n_{1}-m_{1}}\right)^{k_{2}}\left(1+\alpha^{n_{2}-m_{2}}\right)^{-k_{1}} .
$$

By a similar argument as before, we get a contradiction. Thus, $e^{\Gamma_{5}^{\prime}} \neq 1$.

Then, we apply Theorem 3 on the left-hand side of the inequalities (44) with the data

$$
\begin{gathered}
t:=4, \quad \eta_{1}:=a, \quad \eta_{2}:=\alpha, \quad \eta_{3}:=1+\alpha^{m_{1}-n_{1}}, \quad \eta_{4}:=1+\alpha^{m_{2}-n_{2}}, \\
b_{1}:=k_{2}-k_{1}, \quad b_{2}:=k_{2} n_{1}-k_{1} n_{2}, \quad b_{3}:=k_{2}, \quad b_{4}:=k_{1} .
\end{gathered}
$$

Together with combining the right-hand side of (87) with the inequalities (77) and (85), Theorem 3 gives

$$
\begin{aligned}
n_{1} & <4.99 \times 10^{15}\left(n_{1}-m_{1}\right)\left(n_{2}-m_{2}\right)\left(\log n_{2}\right) \\
& <2.43 \times 10^{51}\left(\log n_{2}\right)^{4} .
\end{aligned}
$$

In the above we used the facts that

$$
\min _{1 \leq i \leq 2}\left\{n_{i}-m_{i}\right\}<1.62 \times 10^{11} \log n_{2} \text { and } \max _{1 \leq i \leq 2}\left\{n_{i}-m_{i}\right\}<3 \times 10^{24}\left(\log n_{2}\right)^{2} .
$$

This was obtained under the assumption that the inequality $(88)$ does not hold. If $(88)$ holds, then so does (89). Thus, we have that inequality $(89)$ holds provided that inequality (85) holds. Otherwise, inequality (84) holds which is a better bound than (89). Hence, conclude that $(89)$ holds in all posibble cases.

By the inequality (65),

$$
\log \rho \leq k_{1} \log \rho \leq n_{1} \log \alpha+\log 5<6.92 \times 10^{50}\left(\log n_{2}\right)^{4} .
$$

By substituting this into 73 we get $n_{2}<3.67 \times 10^{134}\left(\log n_{2}\right)^{10}$, and then, by Lemma 4, with the data $r:=10, P:=3.67 \times 10^{134}, L:=n_{2}$, we get that $n_{2}<3.07 \times 10^{162}$. This immediately gives that $n_{1}<4.76 \times 10^{61}$.

We record what we have proved.

Lemma 9. Let $\left(k_{i}, n_{i}, m_{i}\right)$ be a solution to $X_{k_{i}}=P_{n_{i}}+P_{m_{i}}$, with $3 \leq m_{i}<n_{i}$ for $i \in\{1,2\}$ and $1 \leq k_{1}<k_{2}$, then

$$
\max \left\{k_{1}, m_{1}\right\}<n_{1}<4.76 \times 10^{61}, \quad \text { and } \max \left\{k_{2}, m_{2}\right\}<n_{2}<3.07 \times 10^{162} .
$$




\section{REDUCING THE BOUNDS FOR $n_{1}$ AND $n_{2}$ (II)}

In this section we reduce the bounds for $n_{1}$ and $n_{2}$ given in Lemma 6 to cases that can be computationally treated. For this, we return to the inequalities for $\Gamma_{3}^{\prime}, \Gamma_{4}^{\prime}$ and $\Gamma_{5}^{\prime}$.

7.1. The first reduction (II). We divide through both sides of the inequality (74) by $\left(k_{2}-k_{1}\right) \log \alpha$. We get that

$$
\left|\frac{|\log a|}{\log \alpha}-\frac{k_{2} n_{1}-k_{1} n_{2}}{k_{2}-k_{1}}\right|<\frac{42 n_{2}}{\alpha^{\lambda^{\prime}}\left(k_{2}-k_{1}\right)} \quad \text { with } \quad \lambda^{\prime}:=\min _{1 \leq i \leq 2}\left\{n_{i}-m_{i}\right\} .
$$

We assume that $\lambda^{\prime} \geq 10$. Below we apply Lemma 1 . We put $\tau^{\prime}:=\frac{|\log a|}{\log \alpha}$, which is irrational and compute its continued fraction

$$
\left[a_{0}, a_{1}, a_{2}, \ldots\right]=[1,6,2,1,18,166,1,2,13,1,2,5,1,5,1,2,3,1,1,31,1,3, \ldots]
$$

and its convergents

$$
\left[\frac{p_{0}}{q_{0}}, \frac{p_{1}}{q_{1}}, \frac{p_{2}}{q_{2}}, \ldots\right]=\left[1, \frac{7}{6}, \frac{15}{13}, \frac{22}{19}, \frac{411}{355}, \frac{68248}{58949}, \frac{68659}{59304}, \frac{205566}{177557}, \frac{2741017}{2367545}, \ldots\right] .
$$

Furthermore, we note that taking $N:=3.07 \times 10^{162}$ (by Lemma 9 ), it follows that $q_{296}>N>n_{2}>k_{2}-k_{1}$ and $a(N):=\max \left\{a_{j}: 0 \leq j \leq 296\right\}=a_{189}=1028$.

Thus, by Lemma 1, we have that

$$
\left|\tau^{\prime}-\frac{k_{2} n_{1}-k_{1} n_{2}}{k_{2}-k_{1}}\right|>\frac{1}{1030\left(k_{2}-k_{1}\right)^{2}} .
$$

Hence, combining the inequalities (90) and (91), we obtain

$$
\alpha^{\lambda^{\prime}}<43260 n_{2}\left(k_{2}-k_{1}\right)<4.08 \times 10^{329},
$$

so $\lambda^{\prime} \leq 2661$. This was obtained under the assumption that $\lambda^{\prime} \geq 10$, Otherwise, $\lambda^{\prime}<10<2661$ holds as well.

Now, for each $n_{i}-m_{i}=\lambda^{\prime} \in[1,2661]$ we estimate a lower bound $\left|\Gamma_{4}^{\prime}\right|$, with

$$
\Gamma_{4}^{\prime}=\left(k_{j}-k_{i}\right) \log a+\left(k_{j} n_{i}-k_{i} n_{j}\right) \log \alpha+k_{j} \log \left(1+\alpha^{m_{i}-n_{i}}\right)
$$

given in the inequality 80 , via the same procedure described in Subsection 3.3 (LLL-algorithm). We recall that $\Gamma_{4}^{\prime} \neq 0$.

We apply Lemma 3 with the data:

$$
\begin{gathered}
t:=3, \quad \tau_{1}:=\log a, \quad \tau_{2}:=\log \alpha, \quad \tau_{3}:=\log \left(1+\alpha^{-\lambda^{\prime}}\right), \\
x_{1}:=k_{j}-k_{i}, \quad x_{2}:=k_{j} n_{i}-k_{i} n_{j}, \quad x_{3}:=k_{j} .
\end{gathered}
$$

We set $X:=3.99 \times 10^{163}$ as an upper bound to $\left|x_{i}\right|<13 n_{2}$ for all $i=1,2,3$, and $C:=(20 X)^{5}$. A computer in Mathematica search allows us to conclude, together with the inequality 80 , that

$$
8 \times 10^{-660}<\min _{1 \leq \lambda \leq 2661}\left|\Gamma_{4}^{\prime}\right|<16 n_{2} \alpha^{-\nu^{\prime}}, \quad \text { with } \quad \nu^{\prime}:=\min \left\{n_{i}, n_{j}-m_{j}\right\}
$$

which leads to $\nu^{\prime} \leq 6643$. As we have noted before, $\nu^{\prime}=n_{1}$ (so $n_{1} \leq 6643$ ) or $\nu^{\prime}=n_{j}-m_{j}$.

Next, we suppose that $n_{j}-m_{j}=\nu^{\prime} \leq 6643$. Since $\lambda^{\prime} \leq 2661$, we have

$$
\lambda^{\prime}:=\min _{1 \leq i \leq 2}\left\{n_{i}-m_{i}\right\} \leq 2661 \quad \text { and } \quad \chi^{\prime}:=\max _{1 \leq i \leq 2}\left\{n_{i}-m_{i}\right\} \leq 6643 .
$$

Now, returning to the inequality 86 which involves

$$
\begin{aligned}
\Gamma_{5}^{\prime}:= & \left(k_{2}-k_{1}\right) \log a+\left(k_{2} n_{1}-k_{1} n_{2}\right) \log \alpha \\
& +k_{2} \log \left(1+\alpha^{m_{1}-n_{1}}\right)-k_{1} \log \left(1+\alpha^{m_{2}-n_{2}}\right) \neq 0,
\end{aligned}
$$


we use again the LLL-algorithm to estimate the lower bound for $\left|\Gamma_{5}^{\prime}\right|$ and thus, find a bound for $n_{1}$ that is better than the one given in Lemma 9 .

We distinguish the cases $\lambda^{\prime}<\chi^{\prime}$ and $\lambda^{\prime}=\chi^{\prime}$.

7.2. The case $\lambda^{\prime}<\chi^{\prime}$. We take $\lambda^{\prime} \in[1,2661]$ and $\chi^{\prime} \in\left[\lambda^{\prime}+1,6643\right]$ and apply Lemma 3 with the data:

$$
\begin{aligned}
& t:=4, \quad \tau_{1}:=\log a, \quad \tau_{2}:=\log \alpha, \quad \tau_{3}:=\log \left(1+\alpha^{m_{1}-n_{1}}\right), \tau_{4}:=\log \left(1+\alpha^{m_{2}-n_{2}}\right), \\
& x_{1}:=k_{2}-k_{1}, \quad x_{2}:=k_{2} n_{1}-k_{1} n_{2}, \quad x_{3}:=k_{2}, \quad x_{4}:=-k_{1} .
\end{aligned}
$$

We also put $X:=3.99 \times 10^{163}$ and $C:=(20 X)^{9}$. As before, after a computer search in Mathematica together with the inequality 86, we can confirm that

$$
9.9 \times 10^{-1317}<\min _{\substack{1 \leq \lambda \leq 2661 \\ \lambda+1 \leq \chi \leq 6643}}\left|\Gamma_{5}^{\prime}\right|<20 n_{2} \alpha^{-n_{1}} .
$$

This leads to the inequality

$$
\alpha^{n_{1}}<2.02 \times 10^{1317} n_{2} .
$$

Subsitituting for the bound $n_{2}$ given in Lemma 9, we get that $n_{1} \leq 11948$.

7.3. The case $\lambda^{\prime}=\chi^{\prime}$. In this case, we have

$$
\Lambda_{5}^{\prime}:=\left(k_{2}-k_{1}\right)\left(\log a+\log \left(1+\alpha^{m_{1}-n_{1}}\right)\right)+\left(k_{2} n_{1}-k_{1} n_{2}\right) \log \alpha \neq 0 .
$$

We divide through the inequality 86 by $\left(k_{2}-k_{1}\right) \log \alpha$ to obtain

$$
\left|\frac{\left|\log a+\log \left(1+\alpha^{m_{1}-n_{1}}\right)\right|}{\log \alpha}-\frac{k_{2} n_{1}-k_{1} n_{2}}{k_{2}-k_{1}}\right|<\frac{70 n_{2}}{\alpha^{n_{1}}\left(k_{2}-k_{1}\right)}
$$

We now put

$$
\tau_{\lambda^{\prime}}:=\frac{\left|\log a+\log \left(1+\alpha^{-\lambda^{\prime}}\right)\right|}{\log \alpha}
$$

and compute its continued fractions $\left[a_{0}^{\left(\lambda^{\prime}\right)}, a_{1}^{\left(\lambda^{\prime}\right)}, a_{2}^{\left(\lambda^{\prime}\right)}, \ldots\right]$ and its convergents $p_{0}^{(\lambda)} / q_{0}^{\left(\lambda^{\prime}\right)}, p_{1}^{\left(\lambda^{\prime}\right)} / q_{1}^{\left(\lambda^{\prime}\right)}, p_{2}^{\left(\lambda^{\prime}\right)} / q_{2}^{\left(\lambda^{\prime}\right)}, \ldots$ for each $\lambda^{\prime} \in[1,2661]$. Furthermore, for each case we find an integer $t_{\lambda^{\prime}}$ such that $q_{t_{\lambda^{\prime}}}^{\left(\lambda^{\prime}\right)}>N:=3.07 \times 10^{162}>n_{2}>k_{2}-k_{1}$ and calculate

$$
a(N):=\max _{1 \leq \lambda^{\prime} \leq 2661}\left\{a_{i}^{\left(\lambda^{\prime}\right)}: 0 \leq i \leq t_{\lambda^{\prime}}\right\} .
$$

A computer search in Mathematica reveals that for $\lambda^{\prime}=2466, t_{\lambda^{\prime}}=298$ and $i=295$, we have that $a(N)=a_{295}^{(2466)}=2818130$. Hence, combining the conclusion of Lemma 1 and the inequality (96), we get

$$
\alpha^{n_{1}}<70 \times 2818132 n_{2}\left(k_{2}-k_{1}\right)<1.86 \times 10^{333},
$$

so $n_{1} \leq 2690$. Hence, we obtain that $n_{1} \leq 11948$ holds in all cases $\left(\nu^{\prime}=n_{1}\right.$, $\lambda^{\prime}<\chi^{\prime}$ or $\left.\lambda^{\prime}=\chi^{\prime}\right)$. By the inequality $(65)$, we have that

$$
\log \rho \leq k_{1} \log \rho \leq n_{1} \log \alpha+\log 5<3410 .
$$

By considering the second inequality in $(73)$, we can conclude that $n_{2} \leq 8.17 \times$ $10^{39}\left(\log n_{2}\right)^{2}$, which yields $n_{2}<2.76 \times 10^{44}$, by a simple application of Lemma 4 as before. Below, we summarise the first cycle of our reduction process:

$$
n_{1} \leq 11948 \text { and } n_{2} \leq 2.76 \times 10^{44} .
$$

As in the previous case, from the above, we note that the upper bound on $n_{2}$ represents a very good reduction of the bound given in Lemma 9. Hence, we expect that if we restart our reduction cycle with the new bound on $n_{2}$, then 
we get a better bound on $n_{1}$. Thus, we return to the inequality (48) and take $N:=2.76 \times 10^{44}$. A computer search in Mathematica reveals that

$q_{88}>N>n_{2}>k_{2}-k_{1}$ and $a(N):=\max \left\{a_{i}: 0 \leq i \leq 88\right\}=a_{55}=397$,

from which it follows that $\lambda \leq 738$. We now return to $(92)$ and we put $X:=$ $2.76 \times 10^{44}$ and $C:=(10 X)^{5}$ and then apply the LLL-algorithm in Lemma 3 to $\lambda \in[1,738]$. After a computer search, we get

$$
8.6 \times 10^{-183}<\min _{1 \leq \lambda^{\prime} \leq 738}\left|\Gamma_{4}^{\prime}\right|<16 n_{2} \alpha^{-\nu^{\prime}},
$$

then $\nu^{\prime} \leq 1838$. By continuing under the assumption that $n_{j}-m_{j}=\nu \leq 1838$, we return to $(93)$ and put $X:=2.76 \times 10^{44}, C:=(10 X)^{9}$ and $N:=2.76 \times 10^{44}$ for the case $\lambda^{\prime}<\chi^{\prime}$ and $\lambda^{\prime}=\chi^{\prime}$. After a computer search, we confirm that

$$
8 \times 10^{-365}<\min _{\substack{1 \leq \lambda \leq 738 \\ \lambda+1 \leq \chi \leq 1838}}\left|\Gamma_{5}^{\prime}\right|<6 n_{2} \alpha^{-n_{1}}
$$

gives $n_{1} \leq 3304$, and $a(N)=a_{125}^{(160)}=155013$, leads to $n_{1} \leq 774$. Hence, in both cases $n_{1} \leq 3304$ holds. This gives $n_{2} \leq 4 \times 10^{42}$ by a similar procedure as before, and $k_{1} \leq$.

We record what we have proved.

Lemma 10. Let $\left(k_{i}, n_{i}, m_{i}\right)$ be a solution to $X_{i}=P_{n_{i}}+P_{m_{i}}$, with $3 \leq m_{i}<n_{i}$ for $i=1,2$ and $1 \leq k_{1}<k_{2}$, then

$$
m_{1}<n_{1} \leq 3304, \quad k_{1} \leq 3108 \quad \text { and } \quad n_{2} \leq 4 \times 10^{42} .
$$

7.4. The final reduction (II). Returning back to $(60)$ and $(62)$ and using the fact that $\left(X_{1}, X_{1}\right)$ is the smallest positive solution to the Pell equation (2), we obtain

$$
\begin{aligned}
X_{k} & =\rho^{k}+\varrho^{k}=\left(\frac{\left(X_{1}+Y_{1} \sqrt{d}\right.}{2}\right)^{k}+\left(\frac{X_{1}-Y_{1} \sqrt{d}}{2}\right)^{k} \\
& =\left(\frac{X_{1}+\sqrt{X_{1}^{2} \mp 4}}{2}\right)^{k}+\left(\frac{X_{1}-\sqrt{X_{1}^{2} \mp 4}}{2}\right)^{k}:=R_{k}^{ \pm}\left(X_{1}\right) .
\end{aligned}
$$

Thus, we return to the Diophantine equation $X_{k_{1}}=P_{n_{1}}+P_{m_{1}}$ and consider the equations

$$
R_{k_{1}}^{+}\left(X_{1}\right)=P_{n_{1}}+P_{m_{1}} \quad \text { and } \quad R_{k_{1}}^{-}\left(X_{1}\right)=P_{n_{1}}+P_{m_{1}},
$$

with $k_{1} \in[1,3108], m_{1} \in[3,3304]$ and $n_{1} \in\left[m_{1}+1,3304\right]$.

A computer search in Mathematica on the above equations in (98) shows that there are only finitely many solutions that we list in Table 4 below. We note that

$$
\frac{3+\sqrt{5}}{2}=\left(\frac{1+\sqrt{5}}{2}\right)^{2} \quad \text { and } \quad 2+\sqrt{5}=\left(\frac{1+\sqrt{5}}{2}\right)^{3},
$$

so these come from the same Pell equation with $d=5$. Similarly,

$$
\frac{11+\sqrt{13}}{2}=\left(\frac{3+\sqrt{13}}{2}\right)^{2}, \quad \text { and } \quad \frac{51+7 \sqrt{53}}{2}=\left(\frac{7+\sqrt{53}}{2}\right)^{2}
$$

these also come from the same Pell equation with $d=13$ and $d=53$, respectively. 


\begin{tabular}{|ccccc|}
\hline \multicolumn{5}{|c|}{$R_{k_{1}}^{+}\left(X_{1}\right)$} \\
\hline$k_{1}$ & $X_{1}$ & $Y_{1}$ & $d$ & $\rho$ \\
\hline 2 & 3 & 1 & 5 & $(3+\sqrt{5}) / 2$ \\
2 & 4 & 2 & 3 & $2+\sqrt{3}$ \\
2 & 5 & 1 & 21 & $(5+\sqrt{21}) / 2$ \\
3 & 9 & 1 & 77 & $(9+\sqrt{77}) / 2$ \\
2 & 10 & 4 & 6 & $5+2 \sqrt{6}$ \\
2 & 11 & 3 & 13 & $(11+3 \sqrt{13}) / 2$ \\
2 & 12 & 2 & 35 & $6+\sqrt{35}$ \\
2 & 13 & 1 & 165 & $(13+2 \sqrt{165}) / 2$ \\
3 & 15 & 1 & 221 & $(15+\sqrt{221}) / 2$ \\
2 & 25 & 3 & 69 & $(25+3 \sqrt{69}) / 2$ \\
2 & 44 & 2 & 483 & $22+\sqrt{483}$ \\
2 & 51 & 7 & 53 & $(51+7 \sqrt{53}) / 2$ \\
2 & 88 & 6 & 215 & $44+3 \sqrt{215}$ \\
2 & 2570 & 4 & 412806 & $1285+2 \sqrt{412806}$ \\
\hline
\end{tabular}

\begin{tabular}{|ccccc|}
\hline \multicolumn{4}{c|}{$R_{k_{1}}^{-}\left(X_{1}\right)$} \\
\hline$k_{1}$ & $X_{1}$ & $Y_{1}$ & $d$ & $\rho$ \\
\hline 2 & 1 & 1 & 5 & $(1+\sqrt{5}) / 2$ \\
2 & 2 & 2 & 2 & $1+\sqrt{2}$ \\
2 & 3 & 1 & 13 & $(3+\sqrt{13}) / 2$ \\
2 & 4 & 2 & 5 & $2+\sqrt{5}$ \\
2 & 6 & 2 & 10 & $3+\sqrt{10}$ \\
2 & 7 & 1 & 53 & $(7+\sqrt{53}) / 2$ \\
2 & 8 & 2 & 17 & $4+\sqrt{17}$ \\
2 & 10 & 2 & 26 & $5+\sqrt{26}$ \\
2 & 11 & 5 & 5 & $(11+5 \sqrt{5}) / 2$ \\
2 & 19 & 1 & 365 & $(19+\sqrt{365}) / 2$ \\
2 & 22 & 2 & 122 & $11+\sqrt{122}$ \\
2 & 30 & 2 & 226 & $15+\sqrt{226}$ \\
2 & 58 & 2 & 842 & $29+\sqrt{842}$ \\
2 & 88 & 2 & 1937 & $44+\sqrt{1937}$ \\
2 & 178 & 2 & 7922 & $89+\sqrt{7922}$ \\
2 & 3480 & 2 & 3027601 & $1740+\sqrt{3027601}$ \\
\hline
\end{tabular}

TABLE 4 . Solutions to $R_{k_{1}}^{ \pm}\left(X_{1}\right)=P_{n_{1}}+P_{m_{1}}$

From the above tables, we set each $\rho:=\rho_{t}$ for $t=1,2, \ldots 25$. We then work on the linear forms in logarithms $\Gamma_{1}^{\prime}$ and $\Gamma_{2}^{\prime}$, in order to reduce the bound on $n_{2}$ given in Lemma 10. From the inequality (71), for $(k, n, m):=\left(k_{2}, n_{2}, m_{2}\right)$, we write

$$
\left|k_{2} \frac{\log \rho_{t}}{\log \alpha}-n_{2}+\frac{\log a}{\log \left(\alpha^{-1}\right)}\right|<\left(\frac{6}{\log \alpha}\right) \alpha^{-\left(n_{2}-m_{2}\right)}
$$

for $t=1,2, \ldots 25$.

We put

$$
\tau_{t}:=\frac{\log \rho_{t}}{\log \alpha}, \quad \mu_{t}:=\frac{\log a}{\log \left(\alpha^{-1}\right)} \quad \text { and } \quad\left(A_{t}, B_{t}\right):=\left(\frac{6}{\log \alpha}, \alpha\right) .
$$

We note that $\tau_{t}$ is transcendental by the Gelfond-Schneider's Theorem and thus, $\tau_{t}$ is irrational. We can rewrite the above inequality, 99 as

$$
0<\left|k_{2} \tau_{t}-n_{2}+\mu_{t}\right|<A_{t} B_{t}^{-\left(n_{2}-m_{2}\right)}, \quad \text { for } \quad t=1,2, \ldots, 25 \text {. }
$$

We take $N:=4 \times 10^{42}$ which is the upper bound on $n_{2}$ according to Lemma 10 and apply Lemma 2 to the inequality (100). As before, for each $\tau_{t}$ with $t=1,2, \ldots, 25$, we compute its continued fraction $\left[a_{0}^{(t)}, a_{1}^{(t)}, a_{2}^{(t)}, \ldots\right]$ and its convergents $p_{0}^{(t)} / q_{0}^{(t)}, p_{1}^{(t)} / q_{1}^{(t)}, p_{2}^{(t)} / q_{2}^{(t)}, \ldots$ For each case, by means of a computer search in Mathematica, we find and integer $s_{t}$ such that

$$
q_{s_{t}}^{(t)}>2.4 \times 10^{43}=6 N \quad \text { and } \quad \epsilon_{t}:=|| \mu_{t} q^{(t)}||-N|| \tau_{t} q^{(t)} \mid>0 .
$$

We finally compute all the values of $b_{t}:=\left\lfloor\log \left(A_{t} q_{s t}^{(t)} / \epsilon_{t}\right) / \log B_{t}\right\rfloor$. The values of $b_{t}$ correspond to the upper bounds on $n_{2}-m_{2}$, for each $t=1,2, \ldots, 25$, according to Lemma 2. We record the results of the computations for each $t$ in Table 5. 


\begin{tabular}{lllccc}
\hline$t$ & $\rho_{t}$ & $s_{t}$ & $q_{s_{t}}$ & $\epsilon_{t}>$ & $b_{t}$ \\
\hline 1 & $1+\sqrt{2}$ & 78 & $1.46195 \times 10^{44}$ & 0.1578 & 379 \\
2 & $2+\sqrt{3}$ & 100 & $8.93366 \times 10^{43}$ & 0.3147 & 374 \\
3 & $(1+\sqrt{5}) / 2$ & 82 & $2.96985 \times 10^{43}$ & 0.4479 & 369 \\
4 & $5+2 \sqrt{6}$ & 80 & $3.16032 \times 10^{43}$ & 0.1940 & 372 \\
5 & $3+\sqrt{10}$ & 88 & $4.21425 \times 10^{43}$ & 0.2358 & 373 \\
6 & $(3+\sqrt{13}) / 2$ & 91 & $6.62314 \times 10^{43}$ & 0.0666 & 379 \\
7 & $4+\sqrt{17}$ & 92 & $1.11753 \times 10^{44}$ & 0.2387 & 376 \\
8 & $(5+\sqrt{21}) / 2$ & 73 & $2.44965 \times 10^{43}$ & 0.0400 & 377 \\
9 & $5+\sqrt{26}$ & 98 & $3.23107 \times 10^{43}$ & 0.2333 & 372 \\
10 & $6+\sqrt{35}$ & 83 & $1.87425 \times 10^{44}$ & 0.1172 & 381 \\
11 & $(7+\sqrt{53}) / 2$ & 96 & $1.82440 \times 10^{44}$ & 0.3875 & 376 \\
12 & $(25+3 \sqrt{69}) / 2$ & 80 & $2.40911 \times 10^{43}$ & 0.2013 & 371 \\
13 & $(9+\sqrt{77}) / 2$ & 82 & $2.54747 \times 10^{43}$ & 0.1470 & 373 \\
14 & $11+\sqrt{122}$ & 76 & $4.91937 \times 10^{44}$ & 0.4004 & 380 \\
15 & $(13+2 \sqrt{165}) / 2$ & 86 & $2.61323 \times 10^{43}$ & 0.1664 & 372 \\
16 & $44+3 \sqrt{215}$ & 80 & $3.14146 \times 10^{43}$ & 0.3298 & 371 \\
17 & $(15+\sqrt{221}) / 2$ & 75 & $5.70467 \times 10^{43}$ & 0.4661 & 371 \\
18 & $15+\sqrt{226}$ & 79 & $4.78438 \times 10^{43}$ & 0.4046 & 371 \\
19 & $(19+\sqrt{365}) / 2$ & 78 & $3.05270 \times 10^{43}$ & 0.1985 & 372 \\
20 & $22+\sqrt{483}$ & 75 & $4.19689 \times 10^{43}$ & 0.1559 & 374 \\
21 & $29+\sqrt{842}$ & 87 & $8.14707 \times 10^{44}$ & 0.2964 & 382 \\
22 & $44+\sqrt{1937}$ & 87 & $4.70884 \times 10^{43}$ & 0.1191 & 376 \\
23 & $89+\sqrt{7922}$ & 79 & $2.43413 \times 10^{43}$ & 0.4418 & 369 \\
24 & $1285+2 \sqrt{412806}$ & 85 & $2.22078 \times 10^{45}$ & 0.4501 & 385 \\
25 & $1740+\sqrt{3027601}$ & 77 & $2.33761 \times 10^{44}$ & 0.3352 & 378 \\
\hline & & & & &
\end{tabular}

TABLE 5. First reduction compuation results (II)

By replacing $(k, n, m):=\left(k_{2}, n_{2}, m_{2}\right)$ in the inequality (68), we can write

$$
\left|k_{2} \frac{\log \delta_{t}}{\log \alpha}-n_{2}+\frac{\log \left(a\left(1+\alpha^{-\left(n_{2}-m_{2}\right)}\right)\right)}{\log \left(\alpha^{-1}\right)}\right|<\left(\frac{10}{\log \alpha}\right) \alpha^{-n_{2}},
$$

for $t=1,2, \ldots, 25$. We now put

$\tau_{t}:=\frac{\log \delta_{t}}{\log \alpha}, \quad \mu_{t, n_{2}-m_{2}}:=\frac{\log \left(a\left(1+\alpha^{-\left(n_{2}-m_{2}\right)}\right)\right)}{\log \left(\alpha^{-1}\right)} \quad$ and $\quad\left(A_{t}, B_{t}\right):=\left(\frac{10}{\log \alpha}, \alpha\right)$.

With the above notations, we can rewrite (101) as

$$
0<\left|k_{2} \tau_{t}-n_{2}+\mu_{t, n_{2}-m_{2}}\right|<A_{t} B_{t}^{-n_{2}}, \quad \text { for } \quad t=1,2, \ldots 25 .
$$

We again apply Lemma 2 to the above inequality (102), for

$$
t=1,2, \ldots, 25, \quad n_{2}-m_{2}=1,2, \ldots, b_{t}, \quad \text { with } \quad N:=4 \times 10^{43} .
$$

We take

$$
\epsilon_{t, n_{2}-m_{2}}:=\left\|\mu_{t} q^{\left(t, n_{2}-m_{2}\right)}\right\|-N\left\|\tau_{t} q^{\left(t, n_{2}-m_{2}\right)}\right\|>0
$$

and

$$
b_{t, n_{2}-m_{2}}:=\left\lfloor\log \left(A_{t} q_{s_{t}}^{\left(t, n_{2}-m_{2}\right)} / \epsilon_{t, n_{2}-m_{2}}\right) / \log B_{t}\right\rfloor .
$$

With the help of Mathematica, we obtain that 


\begin{tabular}{c|ccccccccccccc}
\hline$t$ & 1 & 2 & 3 & 4 & 5 & 6 & 7 & 8 & 9 & 10 & 11 & 12 & 13 \\
$b_{t, n_{2}-m_{2}}$ & 398 & 404 & 399 & 413 & 390 & 398 & 401 & 397 & 390 & 413 & 401 & 396 & 396 \\
\hline$t$ & 14 & 15 & 16 & 17 & 18 & 19 & 20 & 21 & 22 & 23 & 24 & 25 & \\
$b_{t, n_{2}-m_{2}}$ & 402 & 393 & 395 & 392 & 401 & 396 & 392 & 400 & 401 & 392 & 414 & 395 & \\
\hline
\end{tabular}

TABLE 6. Final reduction computation results (II)

$$
\max \left\{b_{t, n_{2}-m_{2}}: t=1,2, \ldots, 25 \quad \text { and } \quad n_{2}-m_{2}=1,2, \ldots d_{t}\right\} \leq 414 .
$$

Thus, by Lemma 2, we have that $n_{2} \leq 414$, for all $t=1,2, \ldots, 25$, and by the inequality (66) we also have that $n_{1} \leq n_{2}+4$. From the fact that $\rho^{k} \leq 2 \alpha^{n+1}$, we can conclude that $k_{1}<k_{2} \leq 248$. Collecting everything together, our problem is reduced to search for the solutions for (64) in the following range

$$
1 \leq k_{1}<k_{2} \leq 248, \quad 0 \leq m_{1}<n_{1} \in[3,414] \quad \text { and } \quad 0 \leq m_{2}<n_{2} \in[3,414] .
$$

After a computer search on the equation (64) on the above ranges, we obtained the following solutions, which are the only solutions for the exceptional $d$ cases we have stated in Theorem 2,

For the +4 case:

$$
\begin{array}{ll}
(d=3) \quad X_{1}=4=P_{7}+P_{0}=P_{6}+P_{3}=P_{5}+P_{5} \\
\quad X_{2}=14=P_{11}+P_{5}=P_{10}+P_{8}, \quad X_{3}=52=P_{16}+P_{6} \\
(d=5) \quad X_{1}=3=P_{6}+P_{0}=P_{5}+P_{3}, \quad X_{2}=7=P_{9}+P_{0}=P_{7}+P_{6} \\
X_{3}=18=P_{12}+P_{5} \\
(d=21) \quad X_{1}=5=P_{8}+P_{0}=P_{7}+P_{3}=P_{6}+P_{5}, \\
X_{2}=23=P_{13}+P_{5}=P_{12}+P_{9}, \quad X_{3}=2525=P_{30}+P_{11} .
\end{array}
$$

For the -4 case:

$$
\begin{array}{ll}
(d=2) & X_{1}=2=P_{5}+P_{0}=P_{3}+P_{3}, \quad X_{2}=14=P_{11}+P_{5}=P_{10}+P_{8} \\
(d=5) & X_{1}=1=P_{3}+P_{0}, \quad X_{2}=4=P_{7}+P_{0}=P_{6}+P_{3}=P_{5}+P_{5}, \\
& X_{3}=11=P_{10}+P_{5}=P_{9}+P_{7}, \quad X_{4}=29=P_{14}+P_{3} .
\end{array}
$$

This completes the proof of Theorem 2 .

\section{ACKNOWLEDGEMENTS}

The author was supported by the Austrian Science Fund (FWF) projects: F5510-N26 - Part of the special research program (SFB), "Quasi-Monte Carlo Methods: Theory and Applications" and W1230 - "Doctoral Program Discrete Mathematics". Part of this paper was written when the author visited the Institut de Mathématiques de Bordeaux, Université de Bordeaux, in May 2019. He would like to thank this institution for its hospitality and the fruitful working environment.

\section{REFERENCES}

[1] E. F. Bravo, C. A. Gómez, and F. Luca, X-coordinates of Pell equations as sums of two tribonacci numbers, Period. Math. Hungar. 77 (2) (2018), 175-190.

[2] E. F. Bravo, C. A. Gómez, and F. Luca, Correction to: X-coordinates of Pell equations as sums of two tribonacci numbers, Period. Math. Hungar. 80 (1) (2020), 145-146.

[3] Y. Bugeaud, M. Mignotte, and S. Siksek, Classical Classical and modular approaches to exponential Diophantine equations I. Fibonacci and Lucas perfect powers, Ann. of Math. (2) 163 (2) (2006), 969-1018. 
[4] H. Cohen. Number Theory. Volume I: Tools and Diophantine Equations Graduate Texts in Mathematics 239, Springer, 2007.

[5] M. Ddamulira and F. Luca, On the $x$-coordinates of Pell equations which are $k$-generalized Fibonacci numbers, J. Number Theory 207 (2020), 156-195.

[6] A. Dossavi-Yovo, F. Luca, and A. Togbé, On the $X$-coordinates of Pell equations which are rep-digits, Publ. Math. Debrecen, 88 (2016), 381-399.

[7] A. Dujella and A. Pethö, A generalization of a theorem of Baker and Davenport, Quart. J. Math. Oxford Ser. (2), 49(195) (1998), 291-306.

[8] B. Faye and F. Luca, On the $X$-coordinates of Pell equations which are repdigits, Fibonacci Quart., 56 (1) (2018), 52-62.

[9] S. Gúzman Sánchez and F. Luca, Linear combinations of factorials and s-units in a binary recurrence sequence, Ann. Math. Qué. 38 (2) (2014), 169-188.

[10] B. Kafle, F.Luca, and A. Togbé, On the $x$-coordinates of Pell equations which are Fibonacci numbers II, Colloq. Math. 149 (1) (2017), 75-85.

[11] B. Kafle, F. Luca, and A.Togbé, $x$-Coordinates of Pell equations which are Tribonacci numbers II, Period. Math. Hungar. 79 (2) (2019), 157-167.

[12] B. Kafle, F. Luca, and A. Togbé, $X$-coordinates of Pell equations which are Lucas numbers, Bol. Soc. Mat. Mex. 25 (3) (2019), 481-493.

[13] F. Luca, A. Montejano, L. Szalay, and A. Togbé, On the $x$-coordinates of Pell equations which are Tribonacci numbers, Acta Arith., 179 (1) (2017), 25-35.

[14] F. Luca and A. Togbé, On the $x$-coordinates of Pell equations which are Fibonacci numbers, Math. Scand. 122(1) (2018), 18-30.

[15] E. M. Matveev. An explicit lower bound for a homogeneous rational linear form in the logarithms of algebraic numbers II, Izv. Ross. Akad. Nauk Ser. Mat. 64 (6) (2000), 125180, In Russian. English translation in Izv. Math. 64 (6) (2000), 1217-1269.

[16] S. S. Rihane, M. O. Hernane, and A. Togbé, The $x$-coordinates of Pell equations and Padovan numbers, Turkish J. Math. 43 (1) (2019), 207-223.

Mahadi DDAmulira

Institute of Analysis AND Number TheOry

Graz University of Technology

KOPERNIKUSGASSE 24/II

A-8010 Graz, Austria

E-mail address: mddamulira@tugraz.at; mahadi@aims.edu.gh 